AperTO - Archivio Istituzionale Open Access dell'Università di Torino

\title{
Cultural dissimilarity: Boon or Bane for Technology Diffusion?
}

\section{This is a pre print version of the following article:}

Original Citation:

Availability:

This version is available http://hdl.handle.net/2318/1663498

since 2018-06-27T13:17:28Z

Published version:

DOI:10.1016/j.techfore.2018.03.008

Terms of use:

Open Access

Anyone can freely access the full text of works made available as "Open Access". Works made available under a Creative Commons license can be used according to the terms and conditions of said license. Use of all other works requires consent of the right holder (author or publisher) if not exempted from copyright protection by the applicable law. 


\title{
Cultural dissimilarity: Boon or Bane for Technology Diffusion?
}

\author{
Elena Beretta ${ }^{1}$, Magda Fontana ${ }^{2 *}$ \\ Marco Guerzoni ${ }^{2,3,4}$, Alexander Jordan ${ }^{2}$ \\ ${ }^{1}$ DAUIN, Politecnico di Torino, Turin
}

${ }^{2}$ DESPINA, Big Data Lab, Department of Economics and Statistics, University of Turin, Italy

${ }^{3}$ ICRIOS, Bocconi University, Milan

${ }^{4}$ BRICK, Collegio Carlo Alberto, Turin

ABSTRACT: The paper provides a theoretical model of technology adoption based on the idea that the diffusion of information about a technology depends both on the social structure of the adopters and their degree of assortativity. We propose a framework that - while retaining the core assumptions of epidemic diffusion models - allows for explicit modelling of the social structure via social network and of agents cultural heterogeneity via agent-based simulation. Decision-making takes place in institutional contexts where individual features trigger differentiated imitative responses and societal organization acts as medium on which information flows. The model simulates the diffusion of fertilizers in five Ethiopian villages (Peasant Associations), which differ in both political and relational structures and farmers belong to numerous ethnic and religious groups. Starting from survey data we run a compositional understanding simulation with the aim of reproducing observed diffusion curves on the basis of unobserved individual interactions. By minimizing the divergence from model output and observed diffusion, the exercise of categorical calibration and time series fit identify a set of plausible parameters for each village. Results highlight the importance of cultural dissimilarities to understand the diffusion processes.

KEYWORDS: Technology Diffusion; Cultural dissimilarity; agent-based modeling; network analysis

\section{Introduction}

The paper provides a model of technology adoption based on the idea that the diffusion of a technology is affected by non-economic factors such as the social structure in which the adopters are embedded and their degree of assortativity. Assortativity measures the fact that information acquired by ingroup is considered

\footnotetext{
${ }^{*}$ Corresponding author: Despina, Department of Economics and Statistics 'Cognetti de Martiis', Lungo Doria Siena 100A, 10124, Turin, Italy. Email:magda.fontana@unito.it
} 
more reliable and thus affects adoption choices. The role of social and cultural factors in determining the rate of technology adoption has been largely acknowledged in literature since the seminal work by Katz [1961] and highlighted by recent studies such as Walsh et al. [2017], Guerzoni and Jordan [2016] extensively discussed that the literature does not suggest a mechanism. However, this literature is a collection of very informative anecdotal evidence rather than an encompassing framework to explain how culture might impinge on the diffusion process . For instance, Wellin [1955] discussed the poor diffusion of health technology in Peru, Lee and Ungson [2008] the diffusion of ICT in Korea, Nardon and Aten [2008] the ethanol adoption in Brazil, Trompenaars and Hampden-Turner [1998] the diffusion of portable music players in Western societies, and Guerzoni and Jordan [2016] the diffusion of fertilisers in Ethiopia. This is not true for other factors in the vast literature on diffusion of innovation, in which very often clear mechanisms have been both surmised and tested. A detailed review of the literature, which is beyond the scope of this paper, is very well summarized by [Meade and Islam, 2006], who reviewed not only diffusion in economics but also in similar fields such as marketing and organizational studies. A second and well-aged review is by [Lissoni and Metcalfe, 1993] who, on the contrary are more focus on economics of innovation only. Finally, Geroski [2000]'s review focuses on the modelling techniques employed to to describe the process of innovation diffusion.

Thus, we can identify a gap in the literature of diffusion, which has not managed so far to include in a theoretical framework, the otherwise well-documented impact of culture on diffusion. The paper proposes a framework that, while retaining the core assumptions of epidemic diffusion models, allows for explicit modelling of the social structure via social network and of agents cultural heterogeneity via agent-based simulation. The key assumption is that information acquired by individual belonging to a different group is not considered reliable and in some case it is even ignored. The degree of this effect depends on the homophily of the environment, that we measure with an assortativity parameter.

An ideal case study is the diffusion of chemical fertilisers in Ethiopian Peasant Associations, since chemical fertilisers are a superior technology with very low costs of adoption and in the area we observe a very high degree of religious and ethnic differences. The accurate replication of the observed diffusion curves validate the model and corroborates the idea that including non-economic factors in the analysis of technology diffusion improves the explanatory power of models and widens the scope for policy intervention. The paper is organized as follows: Section 2 presents the theoretical frame to simulate diffusion within social networks. Section 3 presents the data selected from the Ethiopia Rural Household Survey (ERHS) and investigates the cultural composition of the rural networks. Section 4 describes the settings and specifications of the agent-based model and Section 5 analyses the general simulation diffusion patterns of the settings. Section 6 presents the best fitting simulation of each village and Section 8.1 reveals the robustness of our results. Finally, Section 7 closes the paper with a summary and some practical considerations. 


\section{Theoretical background}

The analysis is grounded on Griliches [1957] epidemic diffusion model. Epidemic models historically date back to the investigation of smallpox dissemination by Bernoulli [Zhang et al., 2016]. Among them, the susceptible-infected-recovered (SIR) model and the susceptible-infected-susceptible (SIS) model are the most prominent ones. Both models follow the set of assumption: At the beginning, individuals are characterised by being either susceptible or infected. In the next time step, a currently infected individual has the chance to infect a susceptible individual. Afterwards, if the infection was successful the susceptible individual turns its status to infected and the previously infected individual turns into recovered (SIR) or into susceptible (SIS). In the SIR model, recovered individuals cannot be infected again while individuals can be repeatedly infected in the SIS model [Shakarian et al., 2015, Zhang et al., 2016]. Epidemic models fit well our theoretical and empirical scenario. On the theoretical side, epidemic models assume a population of potential adopters where innovation diffuses via information transmitted by individual contact and is mediated by some measure of individual proximity. In our research, we focus on the adoption of a superior technology that is not yet well known by potential adopters. Differently from traditional epidemic models however we model heterogeneous adopters. Heterogeneity impinges on the ability to trigger imitative behavior, i.e. to spread the contagion. In addition, we expand the original epidemic model by introducing social networks as the medium on which the contagion of information takes place. In our setting, the probability of adoption depends on the cultural similarity of the agents sharing the information Guerzoni and Jordan [2016]. Cultural similarity affects adoption in that agents can exhibit homophilic behaviour, i.e. they might tend to give a higher weight to the behaviour of and the information conveyed by similar others [McPherson et al., 2001, Rogers, 2003]. On the empirical side, the data we use for the study regards relatively small communities where information can be easily assumed to be homogeneously available, potential adopters are geographically close, and the technology to be adopted is unique. Moreover, communities differs according to their cultural composition and to their social organization.

In social networks literature, the degree of homophily might be captured by the concept of assortativity. Its impact on the diffusion of innovation is taken into account mainly along two lines: similar individuals easily share information and agents external to a group are more likely to be the source of novel information, as in the case of bridging cliques [Granovetter, 1973, Rogers, 2003]. In the model, assortativity defines sensitivity to difference. In making the adoption decision, agents can discriminate between information deriving from either similar or different individuals. Precisely, we assume that information acquired by a similar individual is considered more reliable. If there is no assortativity, the information from individual in a different group is ignored. Conceptually, we use assortativity in the sense of of Newman [Newman, 2002, 2003], although in the technical operationalization of the model it works differently. For Newman, 
assortativity is a property of the nodes which impact upon the probability of observing an edge among them. In our case, given a network, assortativity impact on the likelihood that an existed edge is activated. The model is a more general version of Newman' approach, since if we draw the network of activated edge, we end up in the standard Newman definition.The idea of coupling epidemic models [Geroski, 2000] with social networks introduces a mechanism very much similar to [Valente, 1996] framework. However, in Valente the ego-network of an individual is evaluated in order to take the adoption decision, while in our model it is simply a way of information diffusion such as in epidemic models.

The diffusion of fertiliser in Ethiopian Peasant Association fits in the the proposed framework. Firstly, homophily plays a crucial role in Sub-Saharan Africa. Although Ethiopia remained largely untouched by the arbitrary redefinitions of borders that followed the end of the colonial era, the resistance to the restructuring of provinces and regional states as part of the ethno-regional federalism has shown that Ethiopia is still characterized by very cohesive differentiated cultural groups, which results in fragile national identities and in frequent local conflicts [Abbink, 2006]. Secondly, cultural heterogeneity reverberates on social structures, i.e. villages exhibit social arrangements that varies considerably in their organization. Finally, the vast body of literature that deals with the diffusion of technology in agricultural contexts falls short in explaining the variance in adoption rate through the traditional socio-economic or geographical factors. The literature covers farm size [David, 1966, Dadi et al., 2004], tenure [Feder et al., 1985], risk \& uncertainty [Havens and Rogers, 1961, Mansfield, 1961, Dercon and Christiaensen, 2011], distance to market [Sunding and Zilberman, 2001], constraints in supply, credit, labour [Croppenstedt et al., 2003, Dadi et al., 2004, Carlsson et al., 2005] as well as the role of neighbours and development agents [Rogers, 2003, Krishnan and Patnam, 2014]. In section 8.2 we compare our results with relevant literature on agricultural in Ethiopia.

\section{Data selection}

The data selection of five rural villages defined as Peasant Associations (PAs) draws from the Ethiopia Rural Household Survey (ERHS), which originally covers fifteen village over the time span from 1994 to 2009. Focusing on five villages accounts for the necessity to observe the beginning of fertiliser diffusion at almost similar dates to study homogeneous diffusion processes. Ethiopian agriculture employs $85 \%$ of the country's available labour force but its factor productivity lags far behind in comparison to western societies [Diao et al., 2007, AGRA, 2014, CIA, 2015]. In addition, Ethiopian soil quality has been worsening for decades and there is an urgent need to adopt innovative technologies and to develop resilience against reoccurring droughts and famines [UNDP Ethiopia, 2014]. Chemical fertilisers such as DAP and Urea bear the potential to augment yields and have been historically promoted and made available by the current Ethiopian government and its predecessors [Kassa, 2003]. 
The focus is on five rural and remote villages without proper infrastructure (dirt roads, no electricity, no local markets and partially long distances, up to $20 \mathrm{~km}$, to the next market). In these villages, access to agricultural technologies depends heavily on the efforts of the government and hence the set of available technology solutions to fight soil degradation is prescribed by the government that focuses its efforts to promote fertilisers. The five villages have been selected since they experienced the first adoption around to the same year (1994), when the survey has been launched and the cultural composition recorded. This choice have some benefits, since we do not face the problem of the first adopter, we know that the first adoption and the subsequent adoption path occurs under similar conditions and, finally, we could identify the cultural composition of each village precisely at the time of adoption.

Figure 1 depicts the fertiliser diffusion patterns for the five PAs. The left-hand side graph displays diffusion curves referring to the date of occurrence per calendar year. Among the villages the first adoption took place in 1992 and the last adoptions were recorded at the end of the survey in 2009. In order to facilitate the comparison of diffusion dynamics, the graph on the right-hand side sets all graphs to an identical starting point. Each village follows a unique diffusion path displaying a high level of variation in the diffusion curves ranging from the sluggish diffusion of Adado up to the rapid curve of Doma. Geblen and Shumsheha follow a similar path in between the two extremes, while Imdibir combines a flat diffusion curve in the first years with a steep increase later on. Due to the late onset of fertiliser diffusion in Adado in 1996 and the truncation after 2009, we limit our analysis for each village to the first 13 years of fertiliser diffusion to allow comparisons in model fit between the villages. 
Figure 1: Fertiliser Diffusion in selected Peasant Associations

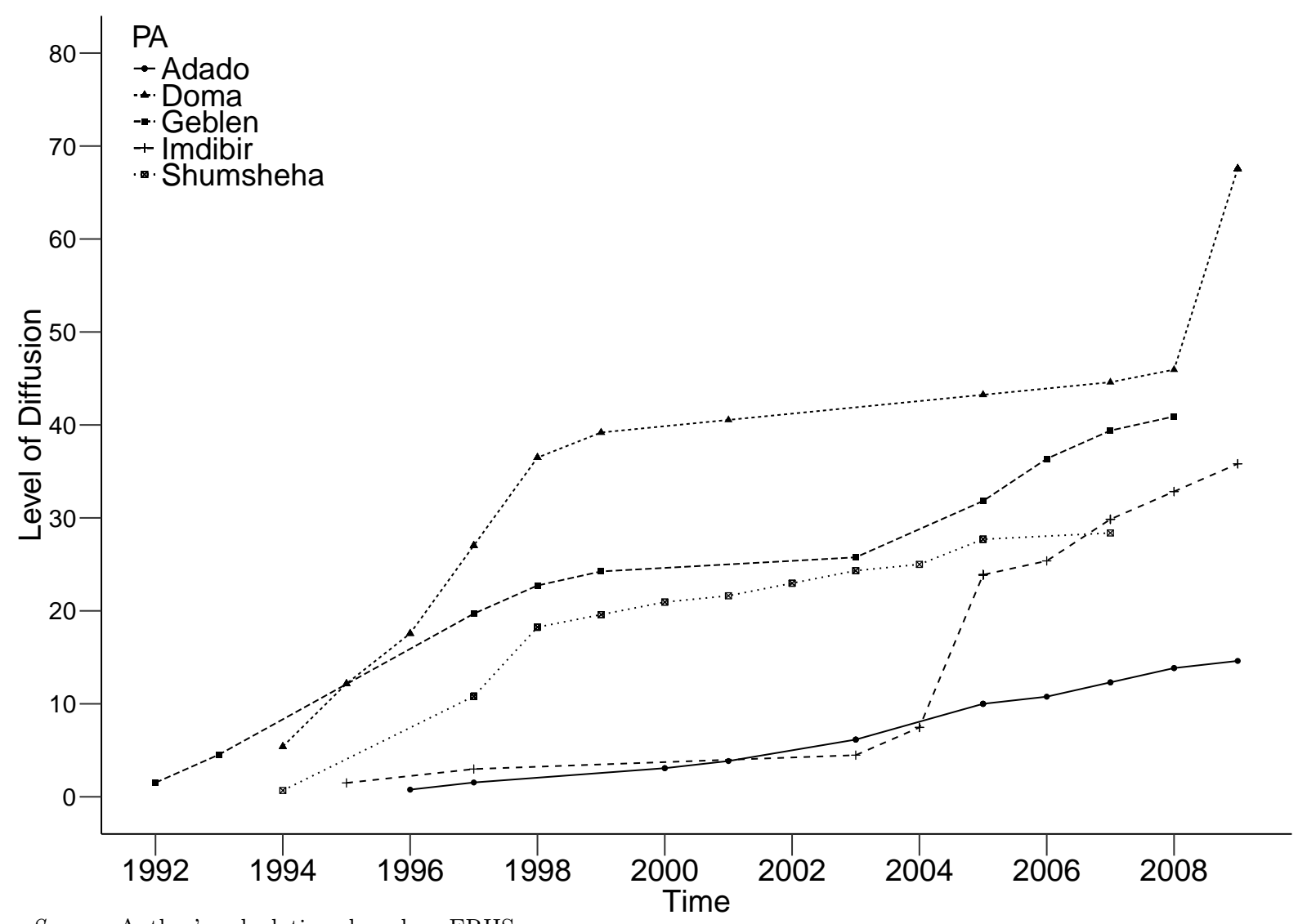

Source: Author's calculations based on ERHS.

A second important information drawn from the survey comprises the ethnicity and the religion of households. Based on this information, we define a cultural group as the unique combination of ethnicity and religious affiliation. The specific combination is set up to account for potentially different interpretations of religious norms in reference to location and ethnicity. The varying interpretations of the same religious faith may result from the restricted overlap with traditional inherent codes of conduct inhabited in ethnicity. Detailed village reports in the survey describe the ongoing presence of various superstitious beliefs in most villages as an expression of the co-existence between religious and ethnic dogmas [Bevan and Pankhurst, 1996a,b,d,e]. Hence, codes of conduct of the rural societies are a mixture of traditional ethnic beliefs and religious norms. This observation is not unique to Ethiopia, instead it is a common phenomenon in SubSaharan Africa where superstitious beliefs are still widespread [Kohnert, 1996, Platteau, 2009, Gershman, 2015, 2016].

Since the Ethiopian society is very much fragmented and for the sake of clarity in the model, we operate a further assumption and classify each cultural group member of the local majority or minority like represented in Figure 2. Figure Figure 2 also displays that the five villages show a large degree of heterogeneity in their cultural composition up to Shumsheha in which the totality of the households belongs to the same cultural 
group. The choice of considering two groups only is based on the idea, that we rather assume that possible discrimination among groups roots in the power structure of the village, rather than in the attitude of a specific ethnicity or religion.

Figure 2: Occurrence of ethnicity and religion by Peasant Association
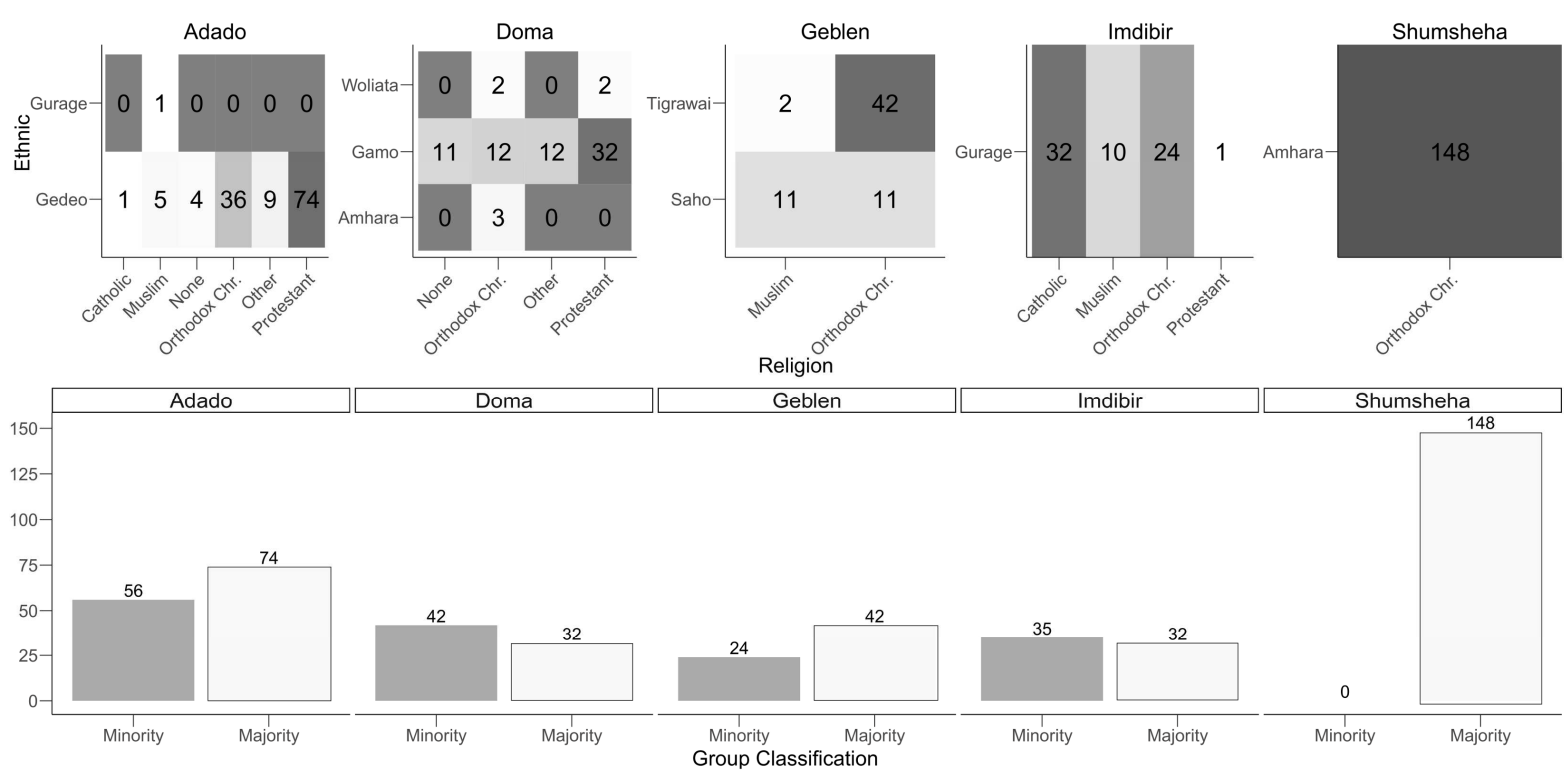

Source: Author's calculations based on ERHS.

Note: Upper figure: co-occurrence of ethnicity and religions of households in the five villages. Lower Figure: classification of households into majority and minority groups.

The analysis of the supplementary Village Studies [Bevan and Pankhurst, 1996a,b,c,d,e] provides quite a comprehensive insight into the history and organisation of each Peasant Association. Reviewing the narrative documents, we extract variables which we use to describe the village and surmise a network structure. This allow us to see whether the surmised structure will correspond to the prediction of the model. In order to avoid a confirmation bias, we assign network structures to village based on the qualitative reading material before the simulation exercise.

The upper part of Table 1 offers an overview about the particular variables that allow the characterization of networks structures for this specific context. The first three attributes are typical examples for preferential attachment networks with their strong dependence on opinion leaders and the crucial role of particular nodes. Importance of friendship and parental relations are characteristics of networks with high clustering. Those close knit networks exert a strong ideological and normative pressure on individuals with mutual control [Bott, 1957]. On the contrary, individuals are more free to deviate from norms and suffer less from social pressure in loose-knit networks as information communication and control are arduous; in fact, loose cultures are generally more tolerant of deviant behaviour than tight cultures, since social norms are weaker[Gelfand and Nishii, 2006]. Frequent migration can induce loose-knit networks occurring among nodes with less intense 
contacts, that connect via weak ties and form less clustered cliques [Granovetter, 1973]. Finally, conflicts occur in dense highly clustered networks but also in structures with strong opinion leaders.

Table 1: Classification according to attributes of network structure

\begin{tabular}{|c|c|c|c|c|}
\hline & \multicolumn{2}{|c|}{ Small-world } & \multicolumn{2}{|c|}{ Preferential attachment } \\
\hline & \multicolumn{2}{|c|}{ Clustering Coefficient } & \multicolumn{2}{|c|}{ Clustering Coefficient } \\
\hline & Low & High & Low & High \\
\hline 1. High importance of elders & & & $\mathrm{X}$ & $\mathrm{X}$ \\
\hline 2. Class stratification & & & $\mathrm{X}$ & $\mathrm{X}$ \\
\hline 3. Tribal, clan or lineage hierarchy & & & $\mathrm{X}$ & $\mathrm{X}$ \\
\hline 4. Importance of friendship & & $\mathrm{X}$ & & $\mathrm{X}$ \\
\hline 5. Importance of parental relations & & $\mathrm{X}$ & & $\mathrm{X}$ \\
\hline 6. Frequent migration & $\mathrm{X}$ & & $\mathrm{X}$ & \\
\hline 7. Ethnic or religious conflict & & $\mathrm{X}$ & $\mathrm{X}$ & $\mathrm{X}$ \\
\hline \multicolumn{5}{|l|}{ Peasant Association } \\
\hline Adado & & & $\mathrm{X}$ & $\mathrm{X}$ \\
\hline Doma & & & & $\mathrm{X}$ \\
\hline Geblen & & & $\mathrm{X}$ & \\
\hline Imdibir & & & & $\mathrm{X}$ \\
\hline Shumsheha & & & $\mathrm{X}$ & $\mathrm{X}$ \\
\hline
\end{tabular}

Note: Classification of network structure attributes and resulting assignment of Peasant Associations towards $\operatorname{network}(\mathrm{s})$.

The lower part of Table 1 displays the assignment of a particular network structure to each village based on the importance of the attributes in the Peasant Associations

\section{Model}

The model simulates the diffusion of a fertiliser in five Peasant Associations. For each PA we run a series of simulations aimed at identifying the set of relevant parameters that best fit the observed diffusion curve.

\subsection{Initialization}

In villages, as well as in many other real world circumstances, individuals differ along multiple dimensions. However, we focus only on whether an individual belongs to the minority and majority line because we do not intend to give a particular meaning (attach a particular behavior or attitude) to religion and ethnicity. This is grounded on a modeling issue and on a moral decision. On the modeling side, having detailed information on which is the precise content or number of differences is not necessary. We assume that individual belonging to different groups might "dislike" others with different intensity (assortativity): they can value the information carried by non- member less than the one carried by members or, to the extreme, they do not want to be like the members of the other(s) group. This applies to a variety of situations that ranges from religious beliefs and ethnicity to fashion or politics, to mention only a few. Majority and 
minority here also hint at a sort of power that the more numerous group can exert on the development of a society when there is conflict between groups. On the moral side, being our empirical application related to religion and ethnicity, the attempt at linking specific groups with given attitudes such as propensity to adopt, is a very delicate endeavor that goes beyond the scope of the paper.

The initialization of the model contains the following assumptions:

(i) Agents are households that, for each PA, are characterised according to the traits expounded in Figure 2 and agents share the same traits if they belong to the same group. The group is defined according to numerosity, hence households belong either to the minority or to the majority.

(ii) Agents can be homophilic in that they might tend to attach more importance to the behaviour of similar agents [Lazarsfeld and Merton, 1954]. Intensity of homophily is described by the assortativity parameter $(0=<q=<1)$. If assortativity is equal to 0 , agents are perfectly homophilic. The assortativity expresses the degree to which households are willing to imitate agents belonging to a different group.

(iii) Minority and majority have separate levels of assortativity $\left(q_{\text {majority }}\right.$ and $\left.q_{\text {minority }}\right)$, i.e. both groups can have different attitudes towards each other.

\subsection{Environment}

Households interact in a network based environment that simulates the (unobserved) social structure of the village. Despite the wide range of existing types of network, the literature that explores real social networked systems is in agreement on the pervasiveness of features like small characteristic path lengths, high clustering coefficients, fat tailed shapes in the degree distributions, degree correlations, and the presence of motifs and community structures [Boccaletti et al., 2006]. In addition, we exclude regular lattices and random graphs because these are homogeneous in interaction (i.e. nodes are topologically equivalent) and therefore do not describe the social scenarios of our interest where the possibility of influencing the neighbors cannot be thought of as being the same across all individuals. We therefore focus on two topologies:

- small world generated with the Kleinberg algorithm;

- preferential attachment generated with the Barabasi-Albert algorithm.

Both networks are simulated with low and high clustering coefficients. For the remainder of the paper, we will refer to the preferential attachment with high clustering when the term hierarchical network is used (Appendix Table 3 provides an overview on our network measures). Small world networks reflect the propensity to create tighter and more numerous relationships with individuals that are close in terms of a given dimension (geographical, religious, political). Cliques are comparable to an inner circle of friends, if one drops out, the relationship between the remaining individuals does not suffer a fragmentation and the nucleus 
remains intact [Granovetter, 1978]. Preferential attachment networks, on the other hand, approximate social interactions mediated by leaders, i.e. nodes with high degree. In societies, a preferential attachment structure, the diffusion process is mediated by a subset of central nodes while diffusion in small world societies results from more decentralized interactions. Appendix Figures 10 - 13 provide exemplary presentations of each network structure.

\subsection{First adopter}

The choice of the seed of the diffusion process within the network aims at appreciating the impact of the (unobserved) individual social relevance on adoption. Social relevance is intended as the degree of the node representing the first adopter. The cultural traits of the first household that adopts the technology are initialized according to data. The model includes three scenarios:

- marginal: chosen with a function that minimizes closeness centrality. The household has a limited social impact, it resides on the fringe of the networks and has a relatively low number of links.

- betweenness: chosen with a function that maximizes betweenness centrality the household is central in the PA, it resides at the cross-roads of social relations.

- eigenvector: chosen with a function that maximizes eigenvector centrality. The household stands in an lower rank in terms of social relevance but is linked with central nodes.

If there exist more than one agent with the same minimum or maximum centrality value than the algorithm randomize among them.

These measures were chosen in order to compare the extremes of the spectrum of influence (marginal vs. betweenness and eigenvector). In order to keep the parameters space within reasonable boundaries and for the sake of synthesis we do not illustrate results for the all intermediate node degree, nevertheless they do not contradict under any respect the presented evidence.

\subsection{Adoption rule}

Each household $i$ decides whether to adopt the technology on the basis of the following stochastic rule:

$$
\operatorname{Adoption}(i, t)= \begin{cases}1, & \text { if } Z<k_{\text {influenceMIN }} \text { or } k_{\text {influenceMAJ }} \\ 0, & \text { otherwise }\end{cases}
$$

with $k_{\text {influenceMIN }}$ when household $i$ belongs to the minority and $k_{\text {influenceMAJ }}$ when household $i$ belongs to the majority. $Z$ assumes a uniformly distributed random value between 0 and 1 . This stochastic process encompasses all the elements that are assumed to affect adoption but that are not explicitly included in the 
model. Neighbours influence $\left(k_{\text {influence }}\right)$ depends on the social environment and of the relationship of the agents inside their neighbourhood. In contrast to linear threshold models, we keep the threshold variable random. However, in line with threshold models the adoption is conditional on the performance of the neighbourhood [Granovetter, 1978]. Agents do not receive external information that could influence their decision to adopt and they rely purely on the dynamics of their environment and their own attitude towards neighbours.. The impact of neighbourhood depends on the cultural group status of a node. For agents from the majority group the neighbourhood influence is given by

$$
k_{\text {influenceMAJ }}=\frac{n_{\text {adopt_majority }}+\left(n_{\text {adopt_minority }} * q_{\text {majority }}\right)}{b}
$$

with $b$ as structure of the social environment quantified by

$$
b=n_{\text {majority }}+\left(n_{\text {minority }} * q_{\text {majority }}\right)
$$

where $n_{\text {majority }}$ and $n_{\text {minority }}$ are the number of neighbours per group, while $n_{\text {adopt_majority }}$ and $n_{\text {adopt_minority }}$ are cumulative group-wise counts of neighbours who have adopted. Thus, the numerator of equation (2a) measures the impact of adopters in their neighbourhood. Imitative behaviour is affected by the level of assortativity, which is map in the parameter $q_{\text {majority }}$ defined between 0 and 1 . Additionally, the denominator, equation (2b), increases the effects of assortativity by weighting the role of neighbours from the minority group.

The effect on adoption of neighbours from the minority group follows the same logic which is summarized in equation (3):

$$
k_{\text {influenceMIN }}=\frac{\left(n_{\text {adopt_majority }} * q_{\text {minority }}\right)+n_{\text {adopt_minority }}}{\left(n_{\text {majority }} * q_{\text {minority }}\right)+n_{\text {minority }}}
$$

\section{Sensitivity analysis}

Table 2: Overview ABM settings

\begin{tabular}{ll}
\hline Worlds & Adado, Doma, Geblen, Imdibir, Shumsheha \\
Networks & hierarchical, preferential-attachment, small-world-high-cluster, small-world-low-cluster \\
Seedings & betweenness, eigenvector, marginal \\
Assortativity Majority & 0 to 1 in 0.1 steps \\
Assortativity Minority & 0 to 1 in 0.1 steps \\
Repetitions & 100 per setting \\
Time Limit & 14 \\
\hline
\end{tabular}

Note: The specific design of each world is available in Appendix Table 4. 
In total we construct 1452 settings for each world, i.e. combination of network type (4), seeding (3) and assortativity levels for majority (11) and minority (11). Since Shumsheha has no cultural variety, applying different assortativity levels would not influence the results and hence we apply a reduced set of twelve combinations. We replicate the simulation of each setting one-hundred times to account for the random component of the model. In order to compare our simulation to the true diffusion we restrict the simulation to 14 time steps (0-13). One time step represents one year, since the information about fertiliser adoption is recorded by year. The simulation produces in total 8,148,000 observations, i.e. 2,032,800 per world with cultural variety and 16,800 observations for Shumsheha.

Simulations in Figure 3 present the impact of network type and seeding method, detached from the assortativity parameters, on the diffusion process in each world. Each unique combination of network and seed displays similar patterns across all worlds. Most apparently, diffusion evolves fastest in small world settings. Scenarios with first adopters in a marginal network position and, in general, the preferential attachment model displays a slower pace of adoption. Apart for the hierarchical network, betweenness and eigenvector seeding differ only marginally and display almost congruent shapes for the other network structures. In the considered time span the typical S-shape diffusion curve occurs only in small world networks with low clustering independently from the seeding method.

Figure 3: Simulation results for all worlds split by type of network
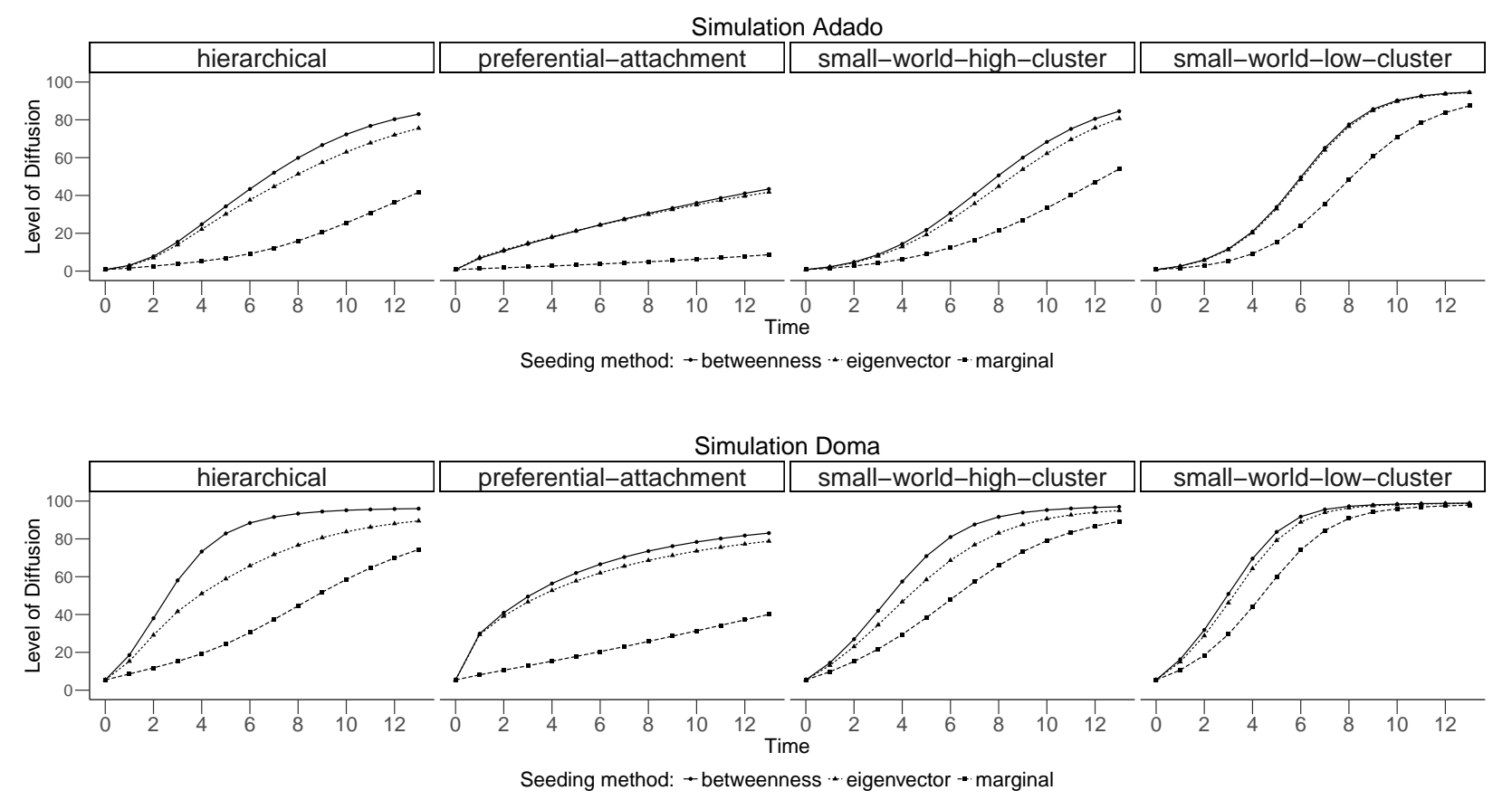


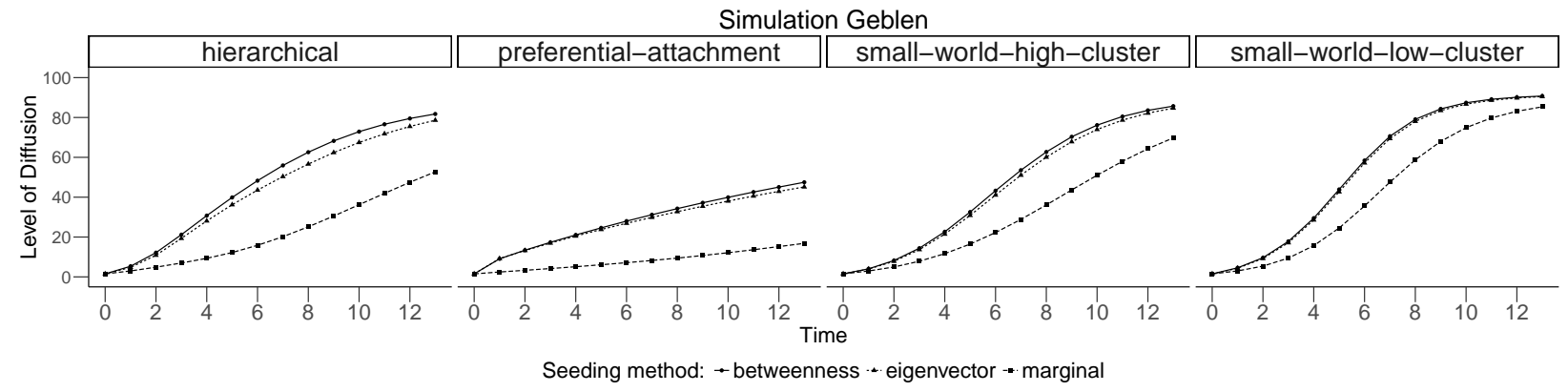

Simulation Shumsheha

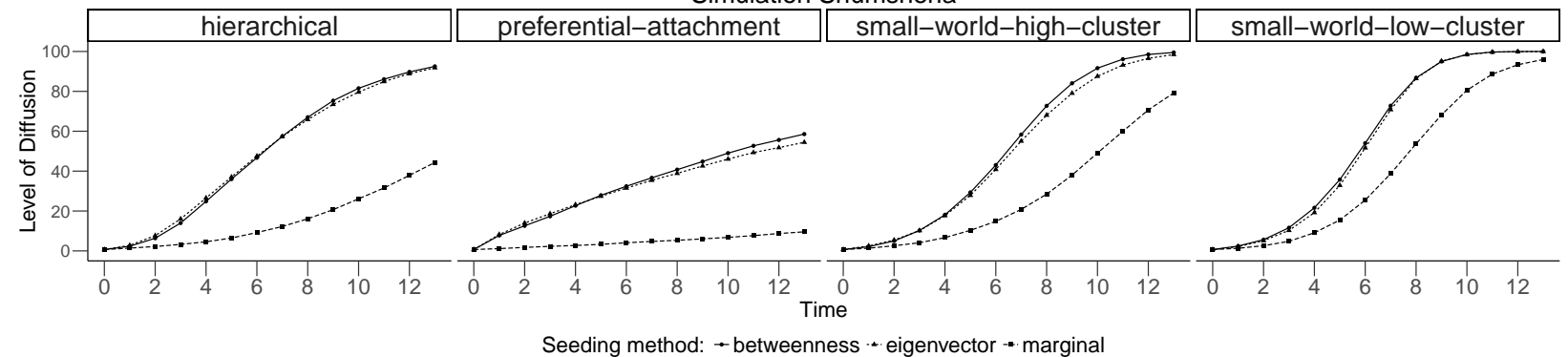

Source: Author's calculations based on ERHS.

Note: Coloured lines represent the diffusion curves by the position of the initial adopter in the network for each graph.

Splitting the above graphs by assortativity levels reveals the influence of group distinctions on the diffusion process (see graph in the Appendix Figures 14 to 18). Hereby, the comparison of the diffusion curves of Geblen (Appendix Figure 16) and Imdibir (Appendix Figure 17) provides a straightforward understanding of the impact of variations in assortativity levels. Both worlds are set up of 66 agents with the first adopter belonging to the minority group. For equal levels of assortativity (especially for low ones), Imdibir presents an accelerated simulated diffusion with respect to Geblen. These differences are driven by the environment of the first adopter. While in Imdibir majority and minority groups are of almost equal size, the majority group in Geblen is $75 \%$ larger than the minority group. Hence, the probability of having members of the same cultural group in the neighbourhood of the first adopter is higher in Imdibir compared to Geblen and as a result, low assortativity levels have a weaker impact on the diffusion process in Imdibir.

Reviewing the simulated diffusion patterns of Doma (Appendix Figure 15) in comparison to the all the other worlds, highlights the importance of multiple first adopters and the advantage of having them instantly in both groups. The combination of both features counteracts the negative impact of low assortativity levels (measured by a value of $q$ close or equal to 1 ) and the technology dissemination evolves faster in general. 


\section{Calibration and Results}

Sensitivity analysis reveals that the most important parameters are the network topology, the position of the first adopter in the network and the levels of assortativity. We therefore conduct a calibration exercise to find parameter values which maximize the fit between the simulation and observed data for each world while the numerosity of cultural groups (minority and majority) is taken from the survey's data. Hence, we compute the absolute difference between diffusion level observations and model results for each time period and sum the difference by run. Hereby, we define the diffusion level for each time step $t$ as fraction of the cumulative number of adopters $\left(n_{\text {adopt }}\right)$ in relation to the absolute quantity of agents/households $\left(n_{\text {agents }}\right)$ per world/village $p$. In more details, based on the model exposed in section 4, we run a series of experiment for each village (with its own numerosity) and all the possible combinations of the above listed parameters. Comparison with observed data is then made for each village (world).

$$
\operatorname{Diffusion}_{p, t}=\frac{\sum_{t=0}^{t} n_{\text {adopt_p,t }}}{n_{\text {agents_p }}}
$$

The best calibration produces the smallest maximum error derived as follows:

$$
e_{i, p}=\sum_{t=0}^{t=13} \mid \text { Diffusion }_{\text {true }, p, t}-\text { Diffusion }_{\text {sim }, p, t, i} \mid
$$

where Diffusion true $, p, t_{\text {is }}$ is the share of real adopters in village $p$ at time $t$ and Diffusion $_{s i m, p, t, i}$ is the share of simulated adopters in world $p$ at time $t$ with the parameter set $i$. The resulting $e_{i, p}$ is the sum over time (0-13) of the absolute deviation of the simulated diffusion level from real diffusion level for each village and parameter set.

Figure 4 shows the best performing setting for each village. Despite the simple model structure with only four variables, the model performs remarkably well to explain the diffusion of the technology in each world (village). 

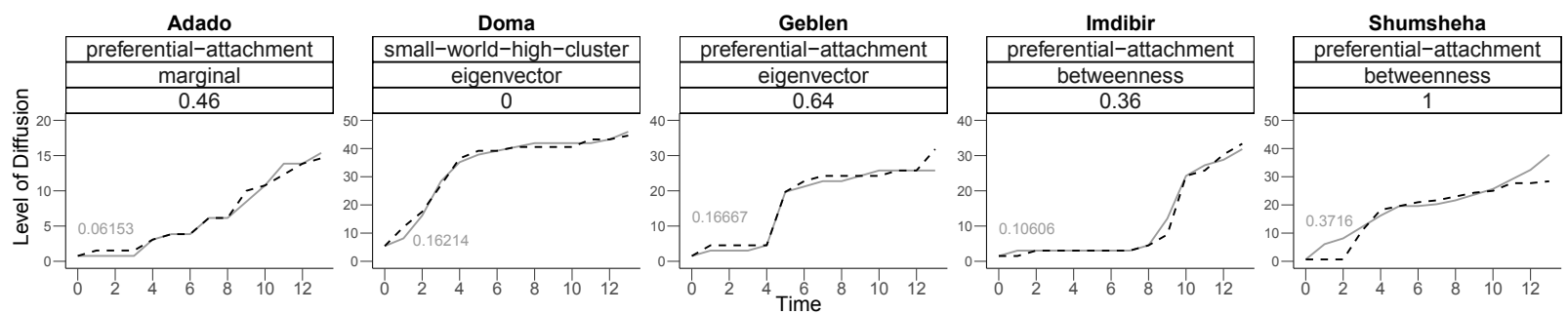

Source: Author's calculations based on ERHS.

Note: Best single simulations out of 145,200 observations per Peasant Association, ordered by deviation from true diffusion. The header of each graph displays the associated settings with network type in the first line, seeding method second and average assortativity in the third line. The coloured number besides the graph reports the corresponding error of the simulation.

The model suggests similar social network structures among the villages but a different position of the first adopter in the network and different levels of the average assortativity.The average assortativity of a world represents the sum of assortativity levels weighted by cultural group shares. From this outcome we can learn few lessons. At the general level, we can argue that in specific situations in which the reality fits the assumptions of an epidemic model, we might describe the process of diffusion in an extremely precise way with a few variables and disregarding the characteristics of the technology. However, in such cases, the network of agents, in which the information diffuses is of a crucial importance. Moreover, the position of the first adopters, although rarely discussed in innovation diffusion literature, plays a crucial role. Finally, we have to highlight that even similar environments, such as rural villages in Ethiopia, exhibit a considerable heterogeneity in these variables. Hence, our considerations only hold true at the general level, if they prove to be robust. For this reason, the next section will show that the best performing parameters as shown in Figure 4 are not random artifacts but they describe a reasonable large set of the best outcomes. We thus show 0.1 percentile with the lowest deviation, we show both single simulations and average results across runs per settings, and average errors for different settings. Finally, we compare the results with the thoroughly qualitative description of each village in the supplementary villages studies.

\section{Concluding remarks}

The paper provides a theoretical model of technology adoption based on the idea that the diffusion of information about a technology depends both on the social structure of the adopters and their degree of assortativity. While the economic determinants of adoption might remain predominant in many cases, this paper shows that adoption in specific circumstances might be better explained by social and cultural drivers. Decision-making takes place in institutional contexts where individual features trigger differentiated imita- 
tive responses and societal organization acts as medium on which information flows. The paper introduces a model of technology diffusion that accounts for both these aspects: Individual heterogeneity and decentralised interaction are modelled through traditional ABM techniques whereas social organization is translated into network topologies. In addition, the position of the first adopter in the network grasps the importance of the individual in the community. The model simulates the diffusion of fertilisers in five Ethiopian villages (Peasant Associations), which differ in both political and relational structures and farmers belong to numerous ethnic and religious groups. Starting from survey data we run a compositional understanding simulation with the aim of reproducing observed diffusion curves on the basis of unobserved individual interactions. The key hypothesis in this application is that assortativity depends on the cultural background of agents which show homophilic behaviour towards similar others in term of religion and ethnicity.

By minimizing the divergence from model output and observed diffusion, the exercise of categorical calibration and time series fit identify a set of plausible parameters for each village.

\subsection{Adoption: heterogeneity matters}

The study builds on traditional epidemic models to investigate the role of heterogeneity in the decision to adopt a superior technology. In the model adoption is driven by imitative behavior at the neighbourhood level and heterogeneity resides in agents' group membership that, in turn, impinges on their ability to trigger imitation. Namely, through the concept of assortativity we introduce sensitivity to difference. In making the adoption decision, agents can discriminate between information deriving from either similar or different individuals. Precisely, we assume that information acquired by a similar individual can be considered more reliable than the one conveyed by a different individual. To the extreme, when there is no assortativity, the information from individuals in a different group is ignored. Assortativity levels vary considerably across communities of adopters when social conflict, status signaling, fashion or membership are taken into account. The application to the diffusion of fertilisers in Ethiopian Peasant Association provides an interesting and fitting example to investigate the role of assortativity. Since Ethiopian villages are characterized by very cohesive differentiated cultural groups that are frequently conflicting, we can therefore assume different levels of assortativity. Results highlight the importance of dissimilarities in understanding adoption since none of the parameter settings replicate the observed diffusion patterns in the absence of cultural diversity.

\subsection{Diffusion: social structure matters}

The analysis is further extended by explicitly modeling the structure of interaction. We assume that information is local and that diffusion also depends on social networks. Adopters are modeled as networks nodes and different topologies are simulated. Small world networks stress the propensity to create tighter and more 
numerous relationships with individuals that are close in terms of a given dimension (geographical, religious, political). Cliques are comparable to an inner circle of friends, if one drops out, the relationship between the remaining individuals does not suffer a fragmentation and the nucleus remains intact. Preferential attachment networks, on the other hand, approximate social interactions mediated by leaders, i.e. nodes with high degree. In societies, a preferential attachment structure, the diffusion process is mediated by a subset of central nodes while diffusion in small world societies results from more decentralized interactions. Results show that small world networks consistently display a higher speed of diffusion than preferential attachment independently of the centrality of the first adopter.

\subsection{Policy implications}

The aim of this study is mainly theoretical, however, our results also provide some policy direction. Although it is rather difficult to intervene in the existing network topology, collecting data on the structure of interaction would improve the estimation of the expected impact of policy that promote innovation. The same holds for the cohesion of the communities: social tensions seems to impact negatively on diffusion independently of the goodness of technology. Development schemes could include assortativity levels in order to identify multiple target that cover the entire span of cultural diversity thereby limiting potentially restrained information flows between groups Krishnan and Patnam [2014]. Finally, our finding point at the importance of nesting the adoption experience in a central network position to promote awareness and to facilitate imitation behaviour in the neighbourhood. Improving data collection in this ambit seems crucial. All these aspects appear particularly evident in the proposed empirical application. Ethiopian policy to promote agricultural innovation heavily relies on extension services [Ragasa et al., 2013]. Development agents do not only provide information but often serve as channel for accessing credits or fertiliser supply [Kassa and Abebaw, 2004]. However, agents are paid according to performance and so naturally target farmers that have the highest probability to repay the debt [Kassa and Abebaw, 2004]. Real adoption curves show that the diffusion process induced by this kind of policy is not entirely satisfactory. Solvent households might not be central in the network or might belong to a non influential group. Changing the incentive schemes for development agents and providing them with information on the the cultural structure of the villages appears to be advisable to spur adoption.

\section{References}

J. Abbink. Ethnicity and Conflict Generation in Ethiopia: Some Problems and Prospects of Ethno-Regional Federalism. Journal of Contemporary African Studies, 24(3):389-413, 2006. 
AGRA. Africa Agriculture Status Report: Climate Change and smallholder agriculture in sub-Saharan Africa. Alliance for a Green Revolution in Africa, Kenya, 2014.

E. N. Banister and M. K. Hogg. Negative symbolic consumption and consumers' drive for self-esteem: The case of the fashion industry. European Journal of Marketing, 38(7):850-868, 2004.

R. H. Bates. When Things Fell Apart: State Failure in Late-Century Africa. Cambridge University Press, 2008.

P. Bevan and A. Pankhurst. Ethiopian Village Studies: Adado. Department of Sociology, Addis Ababa University, Ethiopia and Centre for the Study of African Economies, Oxford, UK, 1996a.

P. Bevan and A. Pankhurst. Ethiopian Village Studies: Doma. Department of Sociology, Addis Ababa University, Ethiopia and Centre for the Study of African Economies, Oxford, UK, 1996b.

P. Bevan and A. Pankhurst. Ethiopian Village Studies: Geblen. Department of Sociology, Addis Ababa University, Ethiopia and Centre for the Study of African Economies, Oxford, UK, 1996c.

P. Bevan and A. Pankhurst. Ethiopian Village Studies: Imdibir. Department of Sociology, Addis Ababa University, Ethiopia and Centre for the Study of African Economies, Oxford, UK, 1996d.

P. Bevan and A. Pankhurst. Ethiopian Village Studies: Shumsheha. Department of Sociology, Addis Ababa University, Ethiopia and Centre for the Study of African Economies, Oxford, UK, 1996e.

S. Boccaletti, V. Latora, Y. Moreno, M. Chavez, and D.-U. Hwang. Complex networks: Structure and dynamics. Physics Reports, 424(4):175 - 308, 2006. ISSN 0370-1573. doi: https://doi.org/10.1016/j.physrep. 2005.10.009. URL http://www.sciencedirect.com/science/article/pii/S037015730500462X.

E. Bott. Family and Social Network: Roles, Norms, and External Relationships in Ordinary Families. London: Tavistock Publications, 1957.

P. Bourdieu. Distinction: A social critique of the judgement of taste. Harvard university press, 1984.

F. Carlsson, G. Köhlin, A. Mekonnen, and M. Yesuf. Are Agricultural Extension Packages what Ethiopian Farmers Want?: A Stated Preference Analysis. 2005.

H. Chun, H. Lee, and D. Kim. The integrated model of smartphone adoption: Hedonic and utilitarian value perceptions of smartphones among korean college students. Cyberpsychology, Behavior, and Social Networking, 15(9):473-479, 2012.

CIA. The World Factbook. Central Intelligence Agency, 2015. URL https://www.cia.gov/library/ publications/the-world-factbook/geos/et.html. (accessed June 11, 2015). 
A. Croppenstedt, M. Demeke, and M. M. Meschi. Technology adoption in the presence of constraints: the case of fertilizer demand in Ethiopia. Review of Development Economics, 7(1):58-70, 2003.

L. Dadi, M. Burton, and A. Ozanne. Duration analysis of technological adoption in Ethiopian agriculture. Journal of Agricultural Economics, 55(3):613-631, 2004.

P. A. David. The Mechanization of Reaping in the Ante-Bellum Midwest. in Henry Rosovsky (ed.), Industrialization in Two Systems: Essays in Honor of Alexander Gerschenkron, pages New York, Wiley and Sons, 3-39, 1966.

S. Dercon and L. Christiaensen. Consumption risk, technology adoption and poverty traps: Evidence from Ethiopia. Journal of Development Economics, 96(2):159-173, Nov 2011.

X. Diao, P. B. Hazell, D. Resnick, and J. Thurlow. The role of agriculture in development: Implications for Sub-Saharan Africa, volume 153. International Food Policy Research Institute, 2007.

G. Feder, R. E. Just, and D. Zilberman. Adoption of Agricultural Innovations in Developing Countries: A Survey. Economic Development and Cultural Change, pages 255-298, 1985.

M. J. Gelfand and L. H. Nishii. On the nature and importance of cultural tightness-looseness. Journal of Applied Psychology, 91(6):1225-1244, 2006.

P. A. Geroski. Models of technology diffusion. Research policy, 29(4):603-625, 2000.

B. Gershman. The economic origins of the evil eye belief. Journal of Economic Behavior $\mathcal{G}$ Organization, 110:119-144, 2015.

B. Gershman. Witchcraft beliefs and the erosion of social capital: Evidence from Sub-Saharan Africa and beyond. Journal of Development Economics, 120:182-208, 2016.

M. Granovetter. Threshold models of collective behavior. American Journal of Sociology, pages 1420-1443, 1978.

M. S. Granovetter. The strength of weak ties. American Journal of Sociology, pages 1360-1380, 1973.

Z. Griliches. Hybrid corn: an exploration in the economics of technical change. Econometrica, 48:501-522, 1957.

M. Guerzoni. Product Variety in Automotive Industry: Understanding Niche Markets in America. Springer Science \& Business Media, 2013.

M. Guerzoni and A. Jordan. "Cursed is the ground because of you": Religion, Ethnicity, and the Adoption of Fertilizers in Rural Ethiopia. "Cognetti di Martiis" Working Paper Series 08/16, 2016. 
M. Guerzoni and M. Nuccio. Music consumption at the dawn of the music industry: the rise of a cultural fad. Journal of cultural economics, 38(2):145-171, 2014.

M. Guerzoni and R. Soellner. Uniqueness seeking and demand estimation in the german automobile industry. Eurasian Business Review, 3(2):179-199, 2013.

A. E. Havens and E. M. Rogers. Adoption of Hybrid Corn: Profitability and Interaction Effects. Rural Sociology, 26(4):409-414, December 1961.

B. Kassa. Agricultural extension in Ethiopia: the case of participatory demonstration and training extension system. Journal of Social Development in Africa, 18(1):49-84, 2003.

B. Kassa and D. Abebaw. Challenges Facing Agricultural Extension Agents: A Case Study from Southwestern Ethiopia. African Development Review, 16(1):139-168, 2004.

E. Katz. The social itinerary of technical change: two studies on the diffusion of innovation. Human Organization, 20(2):70-82, 1961.

D. Kohnert. Magic and Witchcraft: Implications for Democratization and Poverty-Alleviating Aid in Africa. World Development, 24(8):1347-1355, 1996.

P. Krishnan and M. Patnam. Neighbors and extension agents in Ethiopia: Who matters more for technology adoption? American Journal of Agricultural Economics, 96(1):308-327, 2014.

P. F. Lazarsfeld and R. K. Merton. Friendship as a Social Process: A Substantive and Methodological Analysis. Freedom and Control in Modern Society, 18(1):18-66, 1954.

S.-M. Lee and G. R. Ungson. Towards a theory of synchronous technological assimilation: The case of Korea's Internet economy. Journal of World Business, 43(3):274-289, 2008.

F. Lissoni and J. S. Metcalfe. Diffusion of innovation ancient and modern: a review of the main themes. In D. M. Rothwell R., editor, Handbook of Industrial Innovation. Routledge, London, 1993.

E. Mansfield. Technical change and the rate of imitation. Econometrica, 29:741-766, 1961.

M. McPherson, L. Smith-Lovin, and J. M. Cook. Birds of a feather: Homophily in social networks. Annual Review of Sociology, 27(1):415-444, 2001.

N. Meade and T. Islam. Modelling and forecasting the diffusion of innovation-a 25-year review. International Journal of forecasting, 22(3):519-545, 2006.

L. Nardon and K. Aten. Beyond a better mousetrap: a cultural analysis of the adoption of ethanol in Brazil. Journal of World Business, 43(3):261-273, 2008. 
M. E. Newman. Assortative mixing in networks. Physical review letters, 89(20):208701, 2002.

M. E. Newman. Mixing patterns in networks. Physical Review E, 67(2):026126, 2003.

Y. Papanastasiou. Fake news propagation and detection: A sequential model. History, 2017.

R. A. Peterson and A. Simkus. Seven how musical tastes mark occupational status groups. Cultivating differences: Symbolic boundaries and the making of inequality, 152, 1992.

J.-P. Platteau. Institutional obstacles to African economic development: State, ethnicity, and custom. Journal of Economic Behavior \&3 Organization, 71(3):669-689, 2009.

C. Ragasa, G. Berhane, F. Tadesse, and A. S. Taffesse. Gender differences in access to extension services and agricultural productivity. The Journal of Agricultural Education and Extension, 19(5):437-468, 2013.

E. M. Rogers. Diffusion of Innovations. Free Press, New York, 5 edition, 2003.

P. Shakarian, A. Bhatnagar, A. Aleali, E. Shaabani, and R. Guo. Diffusion in Social Networks. Springer, 2015.

G. J. Stigler and G. S. Becker. De gustibus non est disputandum. The american economic review, 67(2): 76-90, 1977.

D. Sunding and D. Zilberman. The agricultural innovation process: research and technology adoption in a changing agricultural sector. Handbook of Agricultural Economics, 1:207-261, 2001.

F. Trompenaars and C. Hampden-Turner. Riding the waves of culture. Citeseer, 1998.

UNDP Ethiopia. National Human Development Report 2014 - Ethiopia: Accelerating Inclusive Growth for Sustainable Human Development in Ethiopia. United Nations Development Programme Ethiopia, 2014.

T. W. Valente. Social network thresholds in the diffusion of innovations. Social networks, 18(1):69-89, 1996.

S. Walsh, Y. Marinakis, R. Harms, and S. Ahluwalia. Explaining Product Adoption and Diffusion at the Base of the Pyramid. International Journal of Technology Intelligence and Planning, forthcoming, 2017.

E. Wellin. Water Boiling in a Peruvian Town, chapter In: "Health, Culture and Community", edited by Benjamin D. Paul, pages 71-103. New York: Russell Sage Foundation, 1955.

Z.-K. Zhang, C. Liu, X.-X. Zhan, X. Lu, C.-X. Zhang, and Y.-C. Zhang. Dynamics of information diffusion and its applications on complex networks. Physics Reports, 651:1-34, 2016. 


\section{Appendix}

\section{Robustness Check}

\section{$8.1 \quad 0.1$ percentile of lowest deviation}

The paragraph presents the best ten single simulations according to the value of $e_{i, p}$ for each village (Figures 5 to 9). In addition to the graphical evaluation, the Appendix tables 6 to 18 provide frequency tables of the sets of parameters for the 0.1 percentile of simulations with the lowest deviation error.

Figure 5: Best single simulations - Adado

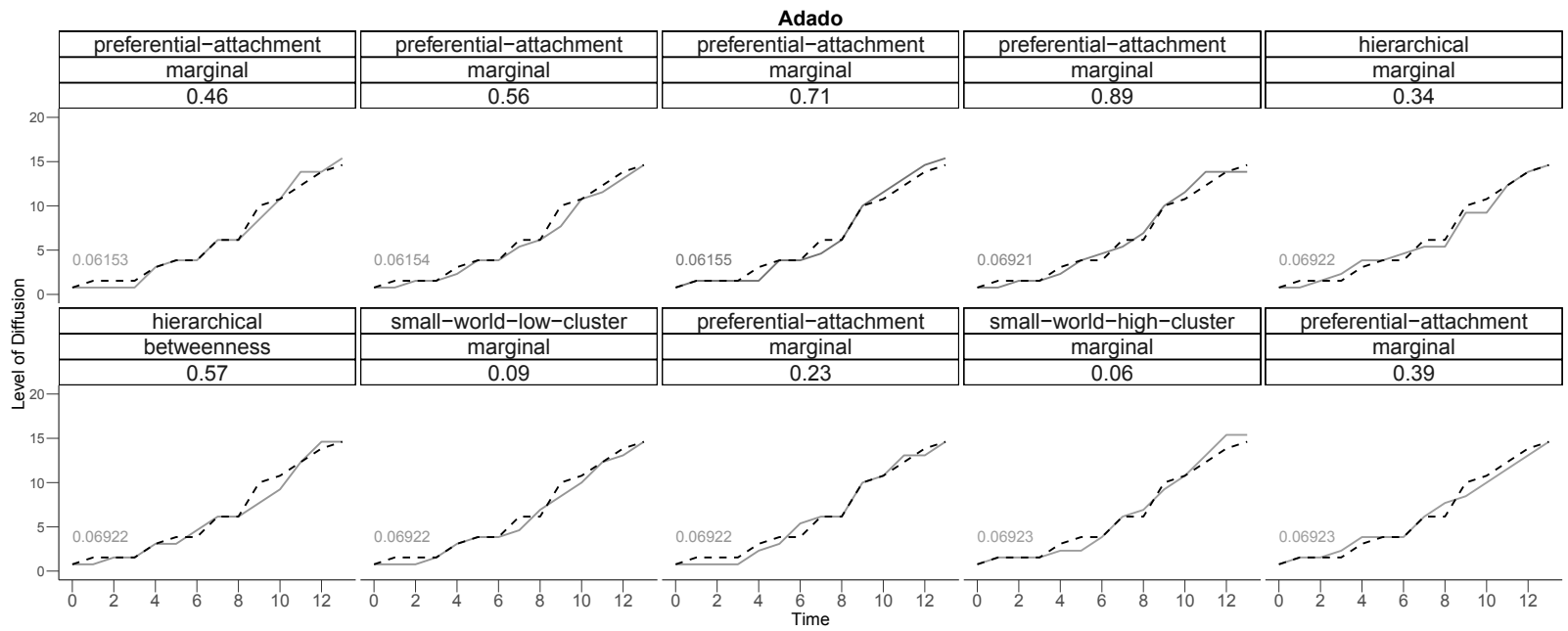

Source: Author's calculations based on ERHS.

Note: Best single simulations out of 145,200 observations, ordered by deviation from true diffusion, starting with the lowest deviation in the top left. The header of each graph displays the associated settings with network type in the first line, seeding method second and average assortativity in the third line. The coloured number besides the graph reports the corresponding error of the simulation. Further corresponding information for Adado is available in the Appendix Tables 5 - 7 .

The diffusion process in the village of Adado is very well described by a network with a preferential attachment, marginal seed and moderate values of average assortativity. Broadening the perspective to the 0.1 percentile of simulations with the lowest error (146 out of 145,200 observations), confirms the preferential attachment network and marginal seeding as best predictions. Interestingly, small world with high clustering occurs only once in Figure 5, but is second in frequency among the $0.1 \%$ quantile results and almost outnumbers preferential attachment. However, these observations go along with in an increase in deviation values and are less precise predictions of the true diffusion in Adado. Finally, the average assortativity of the system displays moderate values with a non-homophilous majority group but quite homophilic members of the minority (Appendix Table 7). 
Figure 6: Doma: Best single simulations

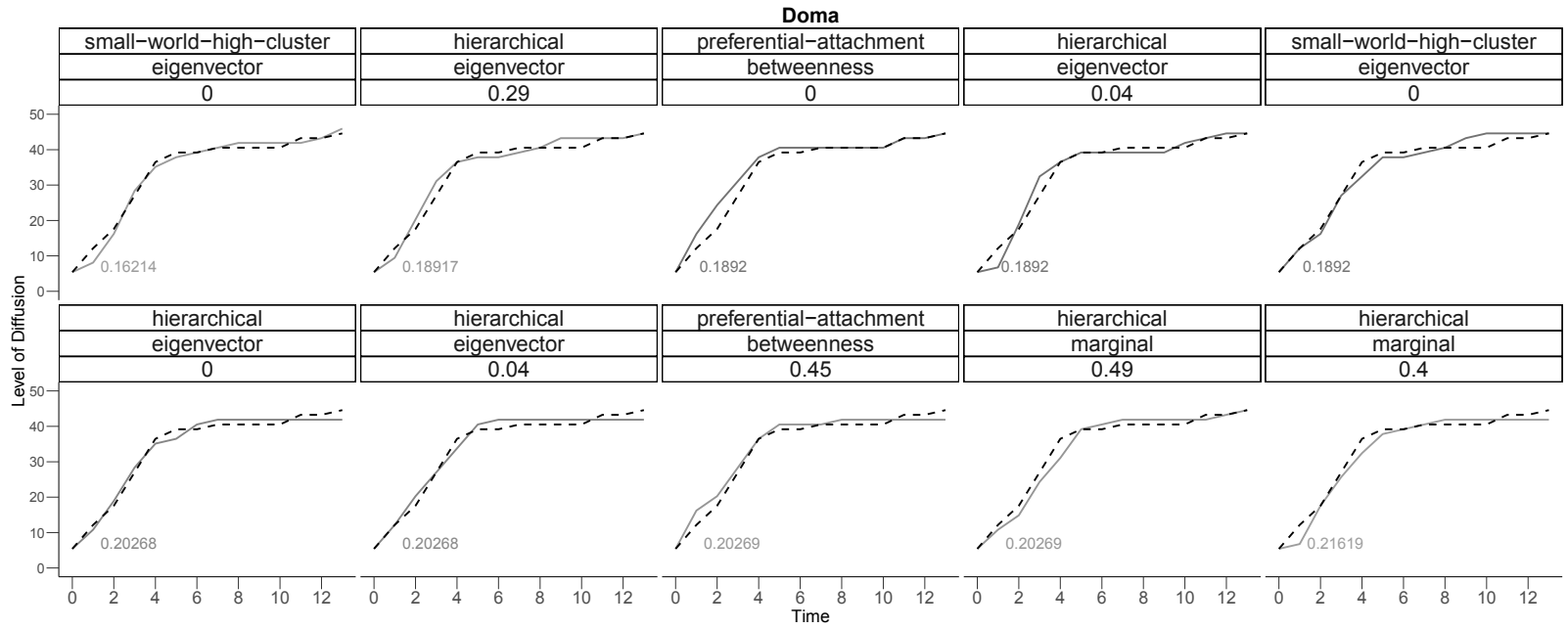

Source: Author's calculations based on ERHS.

Note: Best single simulations out of 145,200 observations, ordered by deviation from true diffusion, starting with the lowest deviation in the top left. The header of each graph displays the associated settings with network type in the first line, seeding method second and average assortativity in the third line. The coloured number besides the graph reports the corresponding error of the simulation. Further corresponding information for Doma is available in the Appendix Tables 8 - 10.

Doma is the only village with multiple first adopters in both groups and a comparable fast take-off in diffusion. In addition, the accumulation of members from the minority group surpasses the total amount of majority group members. Under these circumstances, the eigenvector seed appears to be the dominant solution with six occurrences in Figure 6 and more than half of the counts in the $0.1 \%$ quantile. However, the best prediction appears for a small world with high clustering, the network structure of preferential attachment and hierarchical account for most of best simulations. Instead, the key element in mimicking the real diffusion behaviour for Doma lies in assortativity. The average assortativity levels are relatively low for the most frequent network - seeding combinations in Appendix Table 10 with slightly higher values for the minority group with preferential attachment and a more tolerant majority group for hierarchical networks. Among the ten best predictions, four settings exhibit an assortativity level of zero for both majority and minority, and further four settings display cases where assortativity is zero for one group. These observations guide the conclusion that the first adopters have occupied a central position (eigenvector or betweenness) in the network but the information flow was limited by the low levels of assortativity. We assume that the network structure is either a small world with high clustering or hierarchical (dominant structure in Figure 6) as the high number of preferential attachment appearances are connected to an increasing deviation from the true diffusion. 
Figure 7: Geblen: Best single simulations

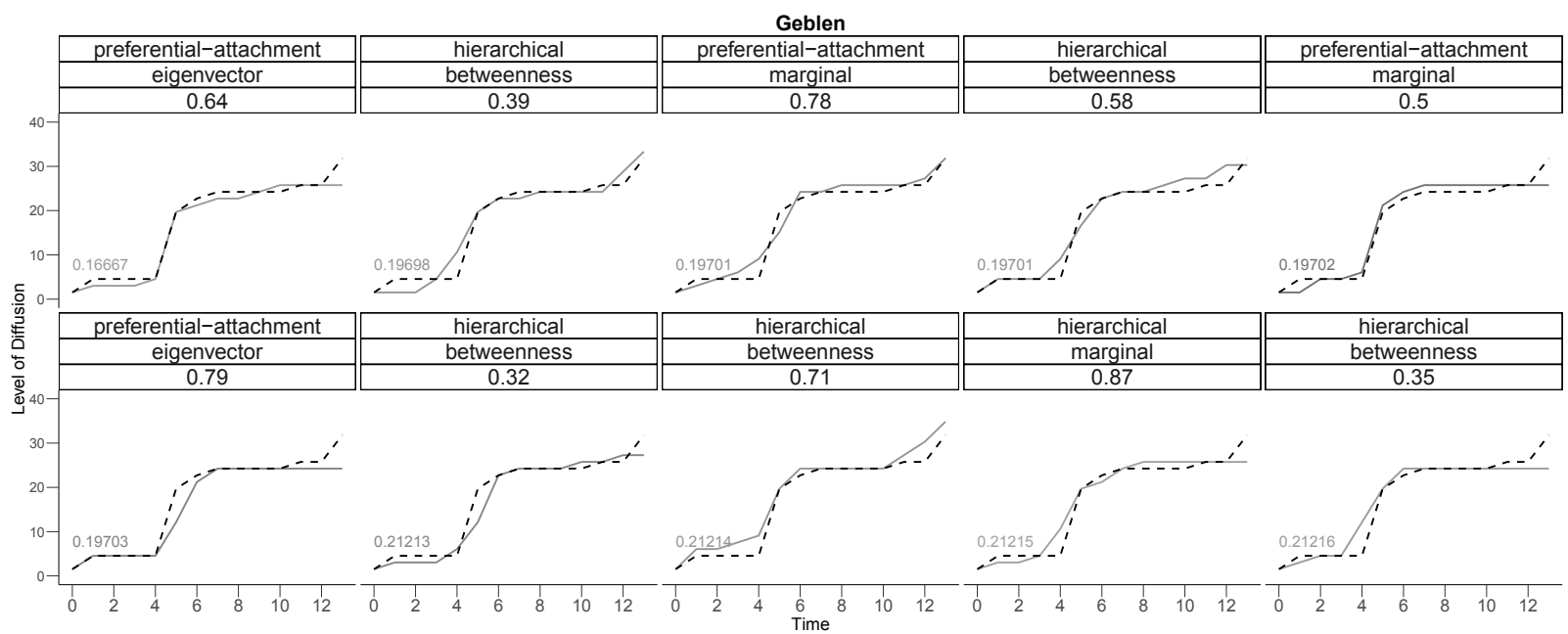

Source: Author's calculations based on ERHS.

Note: Best single simulations out of 145,200 observations, ordered by deviation from true diffusion, starting with the lowest deviation in the top left. The header of each graph displays the associated settings with network type in the first line, seeding method second and average assortativity in the third line. The coloured number besides the graph reports the corresponding error of the simulation. Further corresponding information for Geblen is available in the Appendix Tables 11 - 13.

The simulation results of Geblen clearly shows that the type of network is preferential attachment with either low or high (i.e. hierarchical) clustering. We experience moderate assortativity values for the most frequent network - seeding combinations. Yet, we can neither determine the best network nor the best seeding from Appendix Table 12 with certainty. Several combinations prevail with similar frequency. The position of the first adopter seems to be of minor importance in mimicking the true diffusion as disparity among seedings is not vast. Referring to the result in Figure 7, hierarchical network with betweenness seeding appears to be the most reliable setting. 
Figure 8: Imdibir: Best single simulations

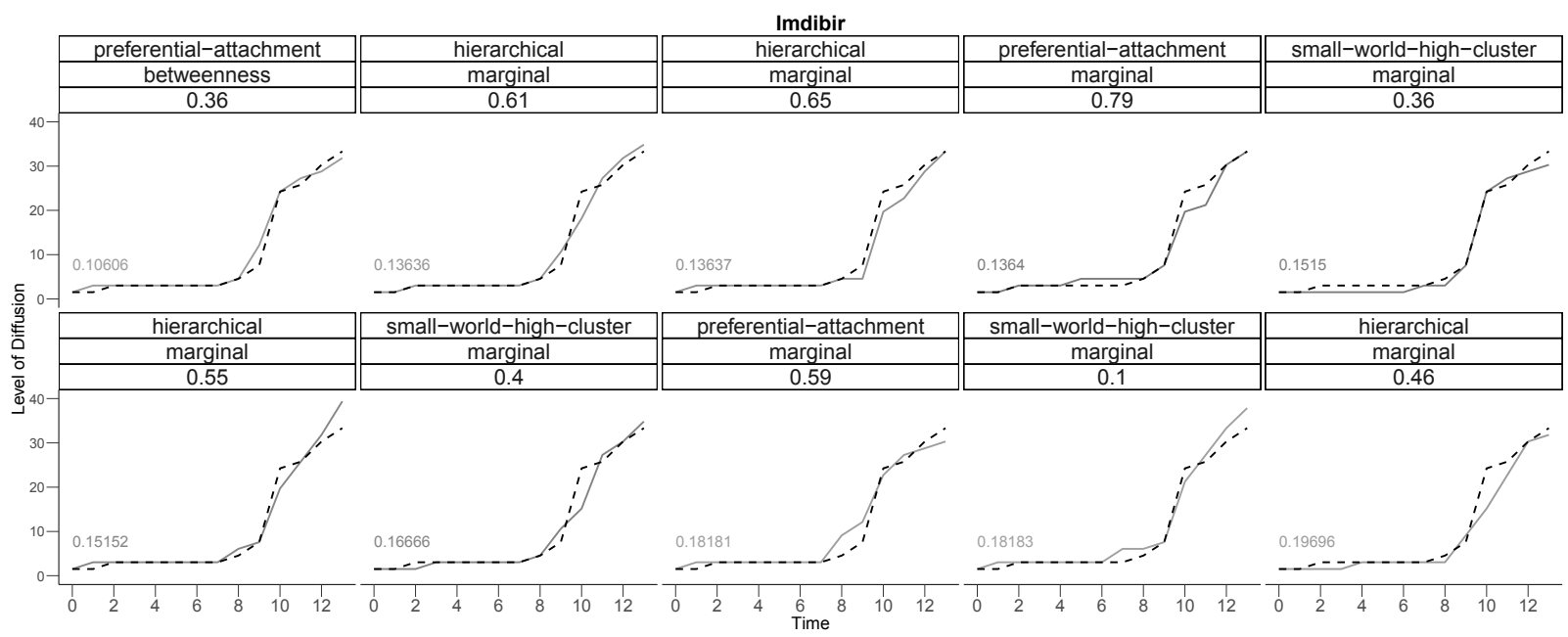

Source: Author's calculations based on ERHS.

Note: Best single simulations out of 145,200 observations, ordered by deviation from true diffusion, starting with the lowest deviation in the top left. The header of each graph displays the associated settings with network type in the first line, seeding method second and average assortativity in the third line. The coloured number besides the graph reports the corresponding error of the simulation. Further corresponding information for Imdibir is available in the Appendix Tables 14 - 16.

For Imdibir, the single setting with the smallest deviation from the true diffusion is preferential attachment with a betweenness seed. However, like in Geblen the extended results are ambiguous. Contrary to the best prediction, it is quite likely that the true first adopter has been in a marginal position as the vast majority of settings in the $0.1 \%$ quantile uses marginal as starting point. The network structure cannot be determined without doubts neither from Figure 8 nor Appendix Tables 14 - 16 as the appearance of small world with high clustering, preferential attachment and hierarchical is almost equally distributed. 
Figure 9: Shumsheha: Best single simulations

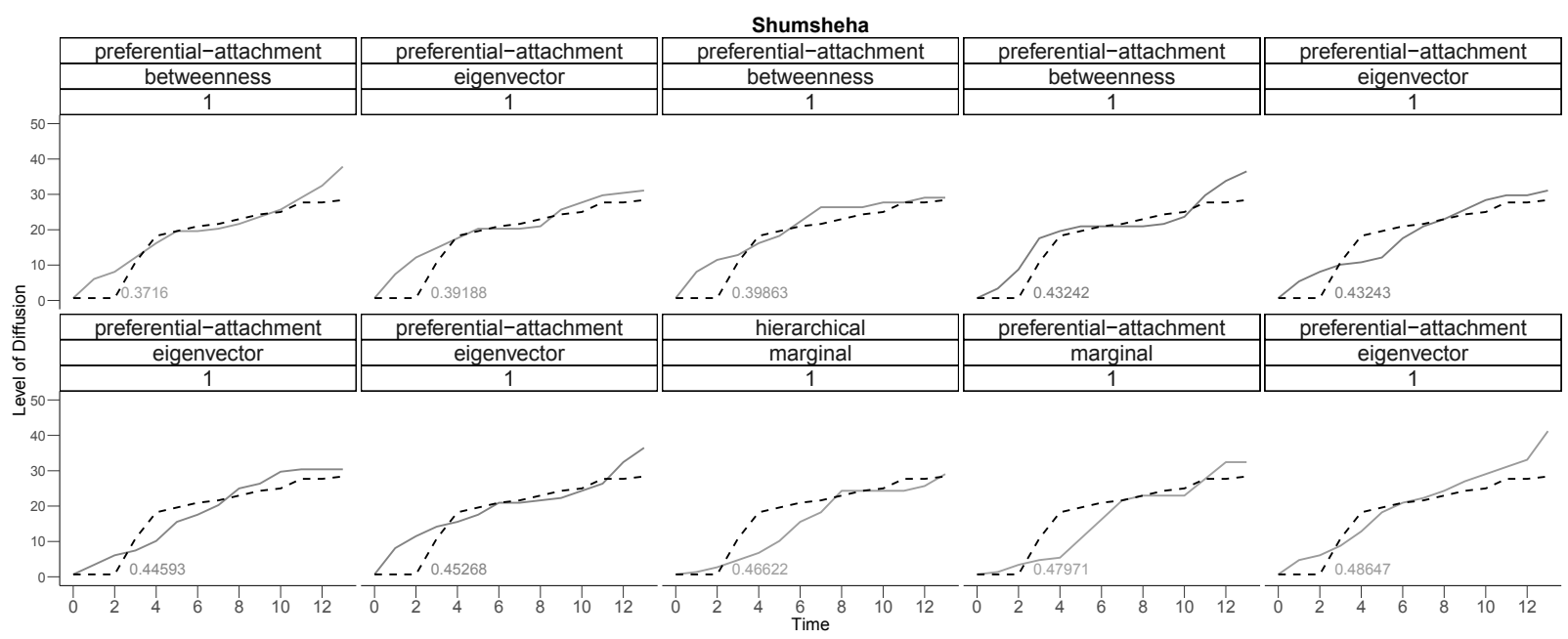

Source: Author's calculations based on ERHS.

Note: Best single simulations out of 1,200 observations, ordered by deviation from true diffusion, starting with the lowest deviation in the top left. The header of each graph displays the associated settings with network type in the first line, seeding method second and average assortativity in the third line. The coloured number besides the graph reports the corresponding error of the simulation. Further corresponding information for Shumsheha is available in the Appendix Tables 17 - 18.

Shumsheha the only Peasant Association without cultural diversity provides a valuable example of the importance of distinctive assortativity levels. Even the best results deviate from true diffusion in roughly double dimension compared to all other simulations above. We achieve the best results with preferential attachment and a non-marginal seed. Since all inhabitants share the same values and beliefs communication should flow without barriers. Yet further veiled factors exist, which influence human interaction and hamper diffusion. A final statement about Shumsheha provides an additional explanation for the missing fit of the simulation to true diffusion process: "[Inhabitants of Shumsheha] generally are, at best, very slow to appropriate new ways of life, including mechanisms of production. This is observed by their refusal to use fertilisers." [Bevan and Pankhurst, 1996e].

\subsection{Revision of supplementary Village Studies}

The Village Studies [Bevan and Pankhurst, 1996a,b,c,d,e] allow us also to interpret the simulation results in the context of conflict or dissimilarities and to suggest an interpretation of the assortativity levels. For this purpose, we need to recall the cultural classification of the Peasant Associations.

In Adado the distinction is driven by religious beliefs rather than ethnicity. Hence, assortativity values below one would hint towards religious frictions. Appendix Table 7 reveals, for all but one settings, a more tolerant (higher assortativity value) attitude of the majority group, i.e. Protestants are more liberal towards the cultural minority than the other way around. Although, the observation would nicely fit the general 
thoughts of Max Weber, the narrative study of Bevan and Pankhurst [1996a] reports a peaceful co-existence of the various religious groups. However, a peaceful co-existence does not explicitly rule out the possibility of subliminal frictions. As a matter of fact, the Village Survey also reports the existence of superstitious beliefs and fear of punishment by witches. These beliefs can eradicate trust [Gershman, 2016] and foster envy [Gershman, 2015]. Therefore, assortativity in Adado may not demarcate religions per se but by their varying affinity to host traditional beliefs and resulting distinctions in trust or tolerance levels.

Just as in Adado, Bevan and Pankhurst [1996b] do not report obvious religious conflicts for Doma. Also superstitious beliefs do not serve as an explanation for the importance of low assortativity levels, due to the missing tolerance of traditional habits by the Protestant church as foremost religious group and the general fading-out of occult beliefs in the Peasant Association. Reviewing our cultural classification with respect to ethnic groups, the low fractionalization may not sufficient to explain the extent of discrimination at first glance, too. Nevertheless, social stratification occurs among both cultural dimensions. In contrast to the Protestant church, other churches do host traditional beliefs and carry out social activities together [Bevan and Pankhurst, 1996b]. Hence, the discrimination between majority and minority recorded by the very low assortativity levels may stem from the lack of common social activities between Protestant and other religions due to the non-overlap of traditional beliefs. Another less subliminal distinction lies in the internal ethnic stratification. Doma is ruled by a caste system with "Pure Gamos" and "Pure Woliatas" on top, with strict prohibitions of particular inter-caste interaction. The Village Survey points the caste system as only source of tension and discrimination in Doma and it is a reasonable explanation for the low assortativity levels in our best simulations.

The relative high levels of assortativity in this context fit narrative of the Village Survey. Bevan and Pankhurst [1996c] report social mixing and inter-marriage between the cultural groups, no prevalent religious or ethnic tensions and no superstitious beliefs. People have close ties due to their common burden of extreme poverty. Nevertheless, other factors apart from cultural distinction are required to justify the assortativity levels below one. A potential explanation may be found in the geographical structure of the Peasant Association. Geblen is located in a mountainous region and households are scattered along the hills, creating communication barriers based on topological distance.

The average assortativity levels in Appendix Table 14 and among the marginal seed in the $0.1 \%$ quantile reveal medium values, hinting towards slight discrimination or disputes. Conflict in mono-ethnical Imdibir can only occur between religious groups. Catholic show less tolerance for the minor religions in nearly all cases (Appendix Table 16). According to Bevan and Pankhurst [1996d] feuds and blood vengeance are frequent and happen on intra- and inter-group level. Additionally, superstitious beliefs are still present. Both appearance together guide the assortativity values away from one.

The lack of cultural diversity in Shumsheha prevents us from properly designing a ABM that fit the 
true diffusion process. The graphical evaluation of of Figure 9 highlights the necessity to identify a barrier for communication flows that would retard the adoption decision and smooth the diffusion process. Like in the other villages apart from Geblen, superstitious beliefs play an important role in the day to day life of Shumsheha's rural society. The associated eradication of trust may be indicated by the previously mentioned unwillingness to accept modern ways of life in Shumsheha [Bevan and Pankhurst, 1996e].

To sum up, the cultural classification serves to explain the role of assortativity in the context of technology diffusion in rural Ethiopia. However, ethnicity and religion just proxy the incorporation of traditional beliefs, which eradicate trust or social cooperation since serious conflicts mostly appear on access to scarce resources and not primarily on cultural stratification [Bates, 2008]. We believe that the model exhibits a considerable degree of generality. While in the current study assortativity depends on religious and ethnic similarity, this parameter can grasp a wide range of differentiated imitative behaviour explaining adoption behaviour, as for instance the belonging to different social classes. This is extremely true for cultural consumption which is considered as a blend of individualist behaviors, imitation, and distinction[Stigler and Becker, 1977, Bourdieu, 1984, Peterson and Simkus, 1992]. Guerzoni and Nuccio [2014] empirically show the imitation effects among social class on music purchasing decisions and highlight the existence of different assortativity for different social groups. However, imitation and distinction dynamics, which can be easily describe within the present social network approach of diffusion with assortativity, go well beyond the case of cultural consumption, since almost each act of purchasing has a cultural component. This is straightforward for the fashion industry [Banister and Hogg, 2004], but also in more traditional one such as the car industry [Guerzoni, 2013, Guerzoni and Soellner, 2013] or the mobile industry [Chun et al., 2012]. Moreover, a contagion approach with assortativity and different groups can also explain difference in virality on the social media of certain information, digital content or fake news among different socio-demographic groups [Papanastasiou, 2017]. 
Table 3: Network measures

\begin{tabular}{|c|c|c|c|c|c|c|c|c|c|c|c|c|c|c|c|c|c|c|c|c|}
\hline \multirow[t]{2}{*}{ PA } & \multicolumn{4}{|c|}{ Max Degree } & \multicolumn{4}{|c|}{ Min Degree } & \multicolumn{4}{|c|}{ Mean path length } & \multicolumn{4}{|c|}{ Density } & \multicolumn{4}{|c|}{ Mean clustering coefficient } \\
\hline & $\begin{array}{c}\text { SmallW } \\
\text { HC }\end{array}$ & $\begin{array}{c}\text { SmallW } \\
\text { LC }\end{array}$ & Pref.Att. & $\begin{array}{l}\text { Hierar- } \\
\text { chical }\end{array}$ & $\begin{array}{c}\text { SmallW } \\
\text { HC }\end{array}$ & $\begin{array}{c}\text { SmallW } \\
\text { LC }\end{array}$ & Pref.Att. & $\begin{array}{l}\text { Hierar- } \\
\text { chical }\end{array}$ & $\begin{array}{c}\text { SmallW } \\
\text { HC }\end{array}$ & $\begin{array}{c}\text { SmallW } \\
\text { LC }\end{array}$ & Pref.Att. & $\begin{array}{c}\text { Hierar- } \\
\text { chical }\end{array}$ & $\begin{array}{c}\text { SmallW } \\
\text { HC }\end{array}$ & $\begin{array}{c}\text { SmallW } \\
\text { LC }\end{array}$ & Pref.Att. & $\begin{array}{l}\text { Hierar- } \\
\text { chical }\end{array}$ & $\begin{array}{l}\text { SmallW } \\
\text { HC }\end{array}$ & $\begin{array}{c}\text { SmallW } \\
\text { LC }\end{array}$ & Pref.Att. & $\begin{array}{l}\text { Hierar- } \\
\text { chical }\end{array}$ \\
\hline Adado & 9 & 8 & 14 & 27 & 3 & 3 & 1 & 5 & 3.895 & 3.148 & 5.715 & 2.75 & 0.041 & 0.043 & 0.015 & 0.085 & 0.16 & 0.03 & 0 & 0.95 \\
\hline Doma & 7 & 8 & 9 & 30 & 3 & 3 & 1 & 2 & 3.55 & 2.96 & 5.128 & 2.366 & 0.062 & 0.066 & 0.027 & 0.19 & 0.25 & 0.03 & 0 & 0.96 \\
\hline Geblen & 7 & 8 & 13 & 26 & 3 & 3 & 1 & 2 & 3.61 & 2.713 & 4.33 & 2.38 & 0.075 & 0.082 & 0.03 & 0.169 & 0.22 & 0.05 & 0 & 0.95 \\
\hline Imdibir & 8 & 8 & 10 & 23 & 3 & 3 & 1 & 3 & 3.3 & 2.68 & 5.53 & 2.8 & 0.078 & 0.083 & 0.03 & 0.2 & 0.17 & 0.06 & 0 & 0.96 \\
\hline Shumsheha & 8 & 9 & 22 & 50 & 3 & 3 & 1 & 7 & 3.65 & 3.3 & 4.62 & 2.44 & 0.035 & 0.036 & 0.013 & 0.15 & 0.09 & 0.03 & 0 & 0.98 \\
\hline
\end{tabular}

Table 4: Overview of the characteristics of each world

\begin{tabular}{|c|c|c|c|c|c|}
\hline & Adado & Doma & Geblen & Imdibir & Shumsheha \\
\hline \multicolumn{6}{|l|}{ Nodes } \\
\hline - Majority & 74 & 32 & 42 & 32 & 148 \\
\hline - Minority & 56 & 42 & 24 & 34 & 0 \\
\hline Total & 130 & 74 & 66 & 66 & 148 \\
\hline \multicolumn{6}{|l|}{ Seed } \\
\hline Size & 1 & 4 & 1 & 1 & 1 \\
\hline \multirow[t]{2}{*}{ Group } & Majority & Majority (2) & Minority & Minority & Majority \\
\hline & & Minority (2) & & & \\
\hline
\end{tabular}

Note: Overview of the characteristics of each world on which we apply settings from Table 2. 
Table 5: Adado: Simulation Results

\begin{tabular}{|c|c|c|c|c|c|c|c|c|c|c|c|}
\hline \multirow[t]{2}{*}{ Error } & \multirow[t]{2}{*}{ Network } & \multirow[t]{2}{*}{ Seed } & \multicolumn{3}{|c|}{ Assortativity } & \multicolumn{3}{|c|}{ Mean } & \multirow[t]{2}{*}{ Density } & \multicolumn{2}{|c|}{ Link Neighbours } \\
\hline & & & Majority & Minority & Average & $\begin{array}{c}\text { Betweenness } \\
\text { Centrality }\end{array}$ & $\begin{array}{l}\text { Clustering } \\
\text { Coefficient }\end{array}$ & $\begin{array}{l}\text { Path } \\
\text { Length }\end{array}$ & & Max & Min \\
\hline 0.06153 & pref.-att. & marginal & 0.2 & 0.8 & 0.46 & 336.638 & 0 & 6.21 & 0.015 & 12 & 1 \\
\hline 0.06154 & pref.-att. & marginal & 0.6 & 0.5 & 0.56 & 284.762 & 0 & 5.41 & 0.015 & 14 & 1 \\
\hline 0.06155 & pref.-att. & marginal & 0.8 & 0.6 & 0.71 & 341.885 & 0 & 6.3 & 0.015 & 9 & 1 \\
\hline 0.06921 & pref.-att. & marginal & 0.8 & 1.0 & 0.89 & 253.246 & 0 & 4.92 & 0.015 & 20 & 1 \\
\hline 0.06922 & hierarchical & marginal & 0.6 & 0.0 & 0.34 & 107.708 & 0.96 & 2.66 & 0.148 & 49 & 1 \\
\hline 0.06922 & hierarchical & betweenness & 1.0 & 0.0 & 0.57 & 161.169 & 0.96 & 3.49 & 0.144 & 29 & 3 \\
\hline 0.06922 & s-w-low-clu. & marginal & 0.0 & 0.2 & 0.09 & 136.692 & 0.03 & 3.11 & 0.043 & 10 & 3 \\
\hline 0.06922 & pref.-att. & marginal & 0.4 & 0.0 & 0.23 & 329.323 & 0 & 6.10 & 0.015 & 10 & 1 \\
\hline 0.06923 & s-w-high-clu. & marginal & 0.1 & 0.0 & 0.06 & 201.331 & 0.19 & 4.12 & 0.040 & 8 & 2 \\
\hline 0.06923 & pref.-att. & marginal & 0.3 & 0.5 & 0.39 & 311.354 & 0 & 5.82 & 0.015 & 15 & 1 \\
\hline
\end{tabular}

Source: Author's calculations based on ERHS.

Note: Corresponding results for Adado Figure 5. Best simulations out of 145,200 observations.

Table 6: Adado: Frequency Table

\begin{tabular}{lccccc}
\hline Seed & \multicolumn{4}{c}{ Network } & $\sum$ \\
\cline { 2 - 5 } & hierarchical & preferential- & small-world- & small-world- & \\
betweenness & 4 & attachment & high-cluster & low-cluster & \\
eigenvector & 2 & 1 & 9 & 1 & 15 \\
marginal & 12 & 1 & 14 & 0 & 17 \\
\hline$\sum$ & 18 & 62 & 34 & 6 & 114 \\
\hline & 18 & 64 & 57 & 7 & 146 \\
\hline
\end{tabular}

Source: Author's calculations based on ERHS.

Note: Frequency of networks and seeding method for the best simulations represented by the $0.1 \%$ quantile for deviation values (146 out of 145,200 observations).
Table 7: Adado: Assortativity

\begin{tabular}{lccccc}
\hline Network & Seed & \multicolumn{3}{c}{ Assortativity } & \multirow{2}{*}{$\begin{array}{c}\text { Average } \\
\text { Error }\end{array}$} \\
\cline { 3 - 5 } & & Average & Majority & Minority & \\
\cline { 3 - 5 } hierarchical & betweenness & 0.55 & 0.95 & 0.02 & 0.08653 \\
hierarchical & eigenvector & 0.53 & 0.90 & 0.05 & 0.08847 \\
hierarchical & marginal & 0.36 & 0.46 & 0.23 & 0.09038 \\
\hline preferential-attachment & betweenness & 0.38 & 0.60 & 0.10 & 0.10000 \\
preferential-attachment & eigenvector & 0.57 & 1 & 0 & 0.09232 \\
preferential-attachment & marginal & 0.61 & 0.58 & 0.64 & 0.09689 \\
\hline small-world-high-cluster & betweenness & 0.36 & 0.58 & 0.05 & 0.09914 \\
small-world-high-cluster & eigenvector & 0.30 & 0.49 & 0.05 & 0.09561 \\
small-world-high-cluster & marginal & 0.29 & 0.40 & 0.13 & 0.09660 \\
\hline small-world-low-cluster & betweenness & 0.57 & 1 & 0 & 0.10772 \\
small-world-low-cluster & marginal & 0.30 & 0.38 & 0.20 & 0.08589 \\
\hline
\end{tabular}

Source: Author's calculations based on ERHS.

Note: Assortativity and average error values for the $0.1 \%$ quantile of deviation values by network and seed. 
Table 8: Doma: Simulation Results

\begin{tabular}{|c|c|c|c|c|c|c|c|c|c|c|c|}
\hline \multirow[t]{2}{*}{ Error } & \multirow[t]{2}{*}{ Network } & \multirow[t]{2}{*}{ Seed } & \multicolumn{3}{|c|}{ Assortativity } & \multicolumn{3}{|c|}{ Mean } & \multirow[t]{2}{*}{ Density } & \multicolumn{2}{|c|}{ Link Neighbours } \\
\hline & & & Majority & Minority & Average & $\begin{array}{c}\text { Betweenness } \\
\text { Centrality }\end{array}$ & $\begin{array}{l}\text { Clustering } \\
\text { Coefficient }\end{array}$ & $\begin{array}{l}\text { Path } \\
\text { Length }\end{array}$ & & Max & Min \\
\hline 0.16214 & s-w-high-clu. & eigenvector & 0.0 & 0.0 & 0.00 & 94.040 & 0.20 & 3.57 & 0.063 & 8 & 2 \\
\hline 0.18917 & hierarchical & eigenvector & 0.4 & 0.2 & 0.29 & 68.459 & 0.94 & 2.87 & 0.158 & 21 & 2 \\
\hline 0.18920 & pref.-att. & betweenness & 0.0 & 0.0 & 0.00 & 159.067 & 0 & 5.35 & 0.027 & 13 & 1 \\
\hline 0.18920 & hierarchical & eigenvector & 0.1 & 0.0 & 0.04 & 56.189 & 0.95 & 2.53 & 0.176 & 24 & 2 \\
\hline 0.18920 & s-w-high-clu. & eigenvector & 0.0 & 0.0 & 0.00 & 102.472 & 0.23 & 3.80 & 0.062 & 7 & 3 \\
\hline 0.20268 & hierarchical & eigenvector & 0.0 & 0.0 & 0.00 & 55.081 & 0.95 & 2.50 & 0.149 & 23 & 5 \\
\hline 0.20268 & hierarchical & eigenvector & 0.1 & 0.0 & 0.04 & 58.621 & 0.94 & 2.60 & 0.167 & 28 & 2 \\
\hline 0.20269 & pref.-att. & betweenness & 0.0 & 0.8 & 0.45 & 162 & 0 & 5.43 & 0.027 & 11 & 1 \\
\hline 0.20269 & hierarchical & marginal & 1.0 & 0.1 & 0.49 & 49.945 & 0.98 & 2.36 & 0.264 & 26 & 4 \\
\hline 0.21619 & hierarchical & marginal & 0.0 & 0.7 & 0.40 & 49.405 & 0.96 & 2.35 & 0.187 & 28 & 2 \\
\hline
\end{tabular}

Source: Author's calculations based on ERHS

Note: Corresponding results for Doma Figure 6. Best simulations out of 145,200 observations.

Table 9: Doma: Frequency Table

\begin{tabular}{lccccc}
\hline Seed & \multicolumn{4}{c}{ Network } & $\sum$ \\
\cline { 2 - 5 } & hierarchical & $\begin{array}{c}\text { preferential- } \\
\text { attachment }\end{array}$ & $\begin{array}{c}\text { small-world- } \\
\text { high-cluster }\end{array}$ & $\begin{array}{c}\text { small-world- } \\
\text { low-cluster }\end{array}$ & \\
\hline betweenness & 1 & 33 & 3 & 0 & 37 \\
eigenvector & 40 & 30 & 13 & 0 & 83 \\
marginal & 9 & 10 & 6 & 1 & 26 \\
\hline$\sum$ & 50 & 73 & 22 & 1 & 146 \\
\hline \hline
\end{tabular}

Source: Author's calculations based on ERHS.

Note: Frequency of networks and seeding method for the best simulations represented by the $0.1 \%$ quantile for deviation values (146 out of 145,200 observations).
Table 10: Doma: Assortativity

\begin{tabular}{lccccc}
\hline Network & Seed & \multicolumn{3}{c}{ Assortativity } & \multirow{2}{*}{$\begin{array}{c}\text { Average } \\
\text { Error }\end{array}$} \\
\cline { 3 - 5 } & & Average & Majority & Minority & \\
\hline hierarchical & betweenness & 0.39 & 0.90 & 0 & 0.28378 \\
hierarchical & eigenvector & 0.22 & 0.28 & 0.18 & 0.27736 \\
hierarchical & marginal & 0.44 & 0.45 & 0.43 & 0.26876 \\
\hline preferential-attachment & betweenness & 0.24 & 0.19 & 0.29 & 0.28951 \\
preferential-attachment & eigenvector & 0.30 & 0.25 & 0.34 & 0.28704 \\
preferential-attachment & marginal & 0.45 & 0.52 & 0.40 & 0.29459 \\
\hline small-world-high-cluster & betweenness & 0.35 & 0.43 & 0.30 & 0.29278 \\
small-world-high-cluster & eigenvector & 0.23 & 0.11 & 0.33 & 0.24428 \\
small-world-high-cluster & marginal & 0.23 & 0.52 & 0.02 & 0.27568 \\
\hline small-world-low-cluster & marginal & 0.09 & 0.20 & 0 & 0.28379 \\
\hline
\end{tabular}

Source: Author's calculations based on ERHS.

Note: Assortativity and average error values for the $0.1 \%$ quantile of deviation values by network and seed. 
Table 11: Geblen: Simulation Results

\begin{tabular}{|c|c|c|c|c|c|c|c|c|c|c|c|}
\hline \multirow[t]{2}{*}{ Error } & \multirow[t]{2}{*}{ Network } & \multirow[t]{2}{*}{ Seed } & \multicolumn{3}{|c|}{ Assortativity } & \multicolumn{3}{|c|}{ Mean } & \multirow[t]{2}{*}{ Density } & \multicolumn{2}{|c|}{ Link Neighbours } \\
\hline & & & Majority & Minority & Average & $\begin{array}{c}\text { Betweenness } \\
\text { Centrality }\end{array}$ & $\begin{array}{l}\text { Clustering } \\
\text { Coefficient }\end{array}$ & $\begin{array}{l}\text { Path } \\
\text { Length }\end{array}$ & & $\operatorname{Max}$ & Min \\
\hline 0.16667 & pref.-att. & eigenvector & 0.6 & 0.7 & 0.64 & 131.197 & 0 & 5.03 & 0.03 & 10 & 1 \\
\hline 0.19698 & hierarchical & betweenness & 0.1 & 0.9 & 0.39 & 47.576 & 0.94 & 2.46 & 0.15 & 21 & 3 \\
\hline 0.19701 & pref.-att. & marginal & 1.0 & 0.4 & 0.78 & 133.227 & 0 & 5.09 & 0.03 & 14 & 1 \\
\hline 0.19701 & hierarchical & betweenness & 0.4 & 0.9 & 0.58 & 44.576 & 0.94 & 2.37 & 0.207 & 26 & 1 \\
\hline 0.19702 & pref.-att. & marginal & 0.5 & 0.5 & 0.50 & 120.652 & 0 & 4.71 & 0.03 & 10 & 1 \\
\hline 0.19703 & pref.-att. & eigenvector & 0.9 & 0.6 & 0.79 & 133.955 & 0 & 5.12 & 0.03 & 10 & 1 \\
\hline 0.21213 & hierarchical & betweenness & 0.1 & 0.7 & 0.32 & 45.273 & 0.95 & 2.39 & 0.186 & 25 & 3 \\
\hline 0.21214 & hierarchical & betweenness & 0.6 & 0.9 & 0.71 & 64.424 & 0.94 & 2.98 & 0.223 & 27 & 1 \\
\hline 0.21215 & hierarchical & marginal & 0.8 & 1.0 & 0.87 & 48.879 & 0.94 & 2.50 & 0.22 & 32 & 1 \\
\hline 0.21216 & hierarchical & betweenness & 0.2 & 0.6 & 0.35 & 61.818 & 0.94 & 2.90 & 0.169 & 19 & 3 \\
\hline
\end{tabular}

Source: Author's calculations based on ERHS.

Note: Corresponding results for Geblen Figure 7. Best simulations out of 145,200 observations.

Table 12: Geblen: Frequency Table

\begin{tabular}{lccccc}
\hline Seed & \multicolumn{4}{c}{ Network } & \multirow{2}{*}{$\sum$} \\
\cline { 2 - 5 } & hierarchical & $\begin{array}{c}\text { preferential- } \\
\text { attachment }\end{array}$ & $\begin{array}{c}\text { small-world- } \\
\text { high-cluster }\end{array}$ & $\begin{array}{c}\text { small-world- } \\
\text { low-cluster }\end{array}$ & \\
\hline betweenness & 23 & 23 & 2 & 9 & 57 \\
eigenvector & 25 & 9 & 5 & 9 & 48 \\
marginal & 12 & 23 & 5 & 0 & 40 \\
\hline$\sum$ & 60 & 55 & 12 & 18 & 145 \\
\hline \hline
\end{tabular}

Source: Author's calculations based on ERHS.

Note: Frequency of networks and seeding method for the best simulations represented by the $0.1 \%$ quantile for deviation values (146 out of 145,200 observations).
Table 13: Geblen: Assortativity

\begin{tabular}{|c|c|c|c|c|c|}
\hline \multirow[t]{2}{*}{ Network } & \multirow[t]{2}{*}{ Seed } & \multicolumn{3}{|c|}{ Assortativity } & \multirow{2}{*}{$\begin{array}{c}\text { Average } \\
\text { Error }\end{array}$} \\
\hline & & Average & Majority & Minority & \\
\hline hierarchical & betweenness & 0.34 & 0.25 & 0.50 & 0.25957 \\
\hline hierarchical & eigenvector & 0.41 & 0.38 & 0.46 & 0.27881 \\
\hline hierarchical & marginal & 0.39 & 0.36 & 0.45 & 0.28033 \\
\hline \multirow{3}{*}{$\begin{array}{l}\text { preferential-attachment } \\
\text { preferential-attachment } \\
\text { preferential-attachment }\end{array}$} & betweenness & 0.54 & 0.58 & 0.46 & 0.27737 \\
\hline & eigenvector & 0.65 & 0.67 & 0.60 & 0.26434 \\
\hline & marginal & 0.61 & 0.67 & 0.49 & 0.25563 \\
\hline \multirow{3}{*}{$\begin{array}{l}\text { small-world-high-cluster } \\
\text { small-world-high-cluster } \\
\text { small-world-high-cluster }\end{array}$} & betweenness & 0.20 & 0 & 0.55 & 0.27275 \\
\hline & eigenvector & 0.23 & 0.18 & 0.32 & 0.26366 \\
\hline & marginal & 0.23 & 0.36 & 0.02 & 0.28184 \\
\hline \multirow{2}{*}{$\begin{array}{l}\text { small-world-low-cluster } \\
\text { small-world-low-cluster }\end{array}$} & betweenness & 0.13 & 0 & 0.36 & 0.27106 \\
\hline & eigenvector & 0.17 & 0 & 0.47 & 0.27780 \\
\hline
\end{tabular}

Source: Author's calculations based on ERHS.

Note: Assortativity and average error values for the $0.1 \%$ quantile of deviation values by network and seed. 
Table 14: Imdibir: Simulation Result

\begin{tabular}{|c|c|c|c|c|c|c|c|c|c|c|c|}
\hline \multirow[t]{2}{*}{ Error } & \multirow[t]{2}{*}{ Network } & \multirow[t]{2}{*}{ Seed } & \multicolumn{3}{|c|}{ Assortativity } & \multicolumn{3}{|c|}{ Mean } & \multirow[t]{2}{*}{ Density } & \multicolumn{2}{|c|}{ Link Neighbours } \\
\hline & & & Majority & Minority & Average & $\begin{array}{l}\text { Betweenness } \\
\text { Centrality }\end{array}$ & $\begin{array}{l}\text { Clustering } \\
\text { Coefficient }\end{array}$ & $\begin{array}{l}\text { Path } \\
\text { Length }\end{array}$ & & Max & Min \\
\hline 0.10606 & pref.-att. & betweenness & 0.1 & 0.6 & 0.36 & 161.015 & 0 & 5.95 & 0.03 & 8 & 1 \\
\hline 0.13636 & hierarchical & marginal & 0.3 & 0.9 & 0.61 & 44.848 & 0.95 & 2.38 & 0.181 & 26 & 2 \\
\hline 0.13637 & hierarchical & marginal & 0.6 & 0.7 & 0.65 & 55.697 & 0.94 & 2.71 & 0.152 & 20 & 2 \\
\hline 0.13640 & pref.-att. & marginal & 1.0 & 0.6 & 0.79 & 103.076 & $\begin{array}{l}.34 \\
0\end{array}$ & 4.17 & 0.03 & 12 & 1 \\
\hline 0.15150 & s-w-high-clu. & marginal & 0.1 & 0.6 & 0.36 & 81.379 & 0.18 & 3.5 & 0.075 & 8 & 2 \\
\hline 0.15152 & hierarchical & marginal & 0.7 & 0.4 & 0.55 & 58.364 & 0.95 & 2.79 & 0.178 & 21 & 2 \\
\hline 0.16666 & s-w-high-clu. & marginal & 0.4 & 0.4 & 0.40 & 73.848 & 0.25 & 3.27 & 0.08 & 8 & 3 \\
\hline 0.18181 & pref.-att. & marginal & 0.9 & 0.3 & 0.59 & 129.485 & 0 & 4.98 & 0.03 & 11 & 1 \\
\hline 0.18183 & s-w-high-clu. & marginal & 0.1 & 0.1 & 0.10 & 76.030 & 0.19 & 3.33 & 0.075 & 7 & 3 \\
\hline 0.19696 & hierarchical & marginal & 0.1 & 0.8 & 0.46 & 59.727 & 0.95 & 2.83 & 0.176 & 21 & 2 \\
\hline
\end{tabular}

Source: Author's calculations based on ERHS

Note: Corresponding results for Imdibir Figure 8. Best simulations out of 145,200 observations.

Table 15: Imdibir: Frequency Table

\begin{tabular}{lccccc}
\hline Seed & \multicolumn{4}{c}{ Network } & $\sum$ \\
\cline { 2 - 5 } & hierarchical & $\begin{array}{c}\text { preferential- } \\
\text { attachment }\end{array}$ & $\begin{array}{c}\text { small-world- } \\
\text { high-cluster }\end{array}$ & $\begin{array}{c}\text { small-world- } \\
\text { low-cluster }\end{array}$ & \\
\hline betweenness & 3 & 7 & 3 & 1 & 14 \\
eigenvector & 6 & 6 & 10 & 6 & 28 \\
marginal & 30 & 26 & 37 & 10 & 103 \\
\hline$\sum$ & 39 & 39 & 50 & 17 & 145 \\
\hline \hline
\end{tabular}

Source: Author's calculations based on ERHS.

Note: Frequency of networks and seeding method for the best simulations represented by the $0.1 \%$ quantile for deviation values (146 out of 145,200 observations).
Table 16: Imdibir: Assortativity

\begin{tabular}{|c|c|c|c|c|c|}
\hline \multirow[t]{2}{*}{ Network } & \multirow[t]{2}{*}{ Seed } & \multicolumn{3}{|c|}{ Assortativity } & \multirow{2}{*}{$\begin{array}{c}\text { Average } \\
\text { Error }\end{array}$} \\
\hline & & Average & Majority & Minority & \\
\hline hierarchical & betweenness & 0.27 & 0.10 & 0.43 & 0.24748 \\
\hline hierarchical & eigenvector & 0.52 & 0.38 & 0.65 & 0.24748 \\
\hline hierarchical & marginal & 0.51 & 0.49 & 0.53 & 0.23789 \\
\hline \multirow{3}{*}{$\begin{array}{l}\text { preferential-attachment } \\
\text { preferential-attachment } \\
\text { preferential-attachment }\end{array}$} & betweenness & 0.41 & 0.27 & 0.54 & 0.21646 \\
\hline & eigenvector & 0.66 & 0.51 & 0.80 & 0.25505 \\
\hline & marginal & 0.67 & 0.69 & 0.66 & 0.24420 \\
\hline \multirow{3}{*}{$\begin{array}{l}\text { small-world-high-cluster } \\
\text { small-world-high-cluster } \\
\text { small-world-high-cluster }\end{array}$} & betweenness & 0.39 & 0.36 & 0.43 & 0.23737 \\
\hline & eigenvector & 0.24 & 0.11 & 0.37 & 0.25455 \\
\hline & marginal & 0.47 & 0.40 & 0.54 & 0.23834 \\
\hline \multirow{3}{*}{$\begin{array}{l}\text { small-world-low-cluster } \\
\text { small-world-low-cluster } \\
\text { small-world-low-cluster }\end{array}$} & betweenness & 0.36 & 0 & 0.70 & 0.27274 \\
\hline & eigenvector & 0.37 & 0 & 0.71 & 0.24497 \\
\hline & marginal & 0.21 & 0.14 & 0.28 & 0.24850 \\
\hline
\end{tabular}

Source: Author's calculations based on ERHS.

Note: Assortativity and average error values for the $0.1 \%$ quantile of deviation values by network and seed 
Table 17: Shumsheha: Simulation Results

\begin{tabular}{|c|c|c|c|c|c|c|c|c|c|c|c|}
\hline \multirow[t]{2}{*}{ Error } & \multirow[t]{2}{*}{ Network } & \multirow[t]{2}{*}{ Seed } & \multicolumn{3}{|c|}{ Assortativity } & \multicolumn{3}{|c|}{ Mean } & \multirow[t]{2}{*}{ Density } & \multicolumn{2}{|c|}{ Link Neighbours } \\
\hline & & & Majority & Minority & Average & $\begin{array}{c}\text { Betweenness } \\
\text { Centrality }\end{array}$ & $\begin{array}{l}\text { Clustering } \\
\text { Coefficient }\end{array}$ & $\begin{array}{c}\text { Path } \\
\text { Length }\end{array}$ & & $\operatorname{Max}$ & Min \\
\hline 0.37160 & pref.-att. & betweenness & 1 & 1 & 1 & 328.17 & 0 & 5.46 & 0.013 & 13 & 1 \\
\hline 0.39188 & pref-att. & eigenvector & 1 & 1 & 1 & 340.15 & 0 & 5.62 & 0.013 & 13 & 1 \\
\hline 0.39863 & pref.-att. & betweenness & 1 & 1 & 1 & 311.57 & 0 & 5.23 & 0.013 & 14 & 1 \\
\hline 0.43242 & pref.-att. & betweenness & 1 & 1 & 1 & 368.24 & 0 & 6.01 & 0.013 & 12 & 1 \\
\hline 0.43243 & pref.-att. & eigenvector & 1 & 1 & 1 & 385.52 & 0 & 6.24 & 0.013 & 11 & 1 \\
\hline 0.44593 & pref.-att. & eigenvector & 1 & 1 & 1 & 353.85 & 0 & 5.81 & 0.013 & 11 & 1 \\
\hline 0.45268 & pref.-att. & eigenvector & 1 & 1 & 1 & 391.32 & 0 & 6.32 & 0.013 & 13 & 1 \\
\hline 0.46622 & hierarchical & marginal & 1 & 1 & 1 & 133.23 & 0.96 & 2.81 & 0.129 & 42 & 5 \\
\hline 0.47971 & pref.-att. & marginal & 1 & 1 & 1 & 328.88 & 0 & 5.47 & 0.013 & 20 & 1 \\
\hline 0.48647 & pref.-att. & eigenvector & 1 & 1 & 1 & 384.37 & 0 & 6.22 & 0.013 & 11 & 1 \\
\hline
\end{tabular}

Source: Author's calculations based on ERHS

Note: Corresponding results for Imdibir Figure 9. Best simulations out of 1,200 observations.

Table 18: Shumsheha: Frequency Table

\begin{tabular}{lccccc}
\hline Seed & \multicolumn{4}{c}{ Network } & $\sum$ \\
\cline { 2 - 5 } & hierarchical & $\begin{array}{c}\text { preferential- } \\
\text { attachment }\end{array}$ & $\begin{array}{c}\text { small-world- } \\
\text { high-cluster }\end{array}$ & $\begin{array}{c}\text { small-world- } \\
\text { low-cluster }\end{array}$ & \\
\hline betweenness & $0(2)$ & $3(35)$ & $0(0)$ & $0(0)$ & $3(37)$ \\
eigenvector & $0(3)$ & $7(42)$ & $0(0)$ & $0(0)$ & $8(45)$ \\
marginal & $1(16)$ & $1(8)$ & $0(14)$ & $0(0)$ & $2(38)$ \\
\hline$\sum$ & $1(21)$ & $11(85)$ & $0(14)$ & $0(0)$ & $12(120)$ \\
\hline \hline
\end{tabular}

Source: Author's calculations based on ERHS.

Note: Frequency of networks and seeding method for the best simulations represented by the $1 \%(10 \%)$ quantile for deviation values (12 (120) out of 1,200 observations). 
Figure 10: Hierarchical network

Hierarchical network with marginal position of first adopter

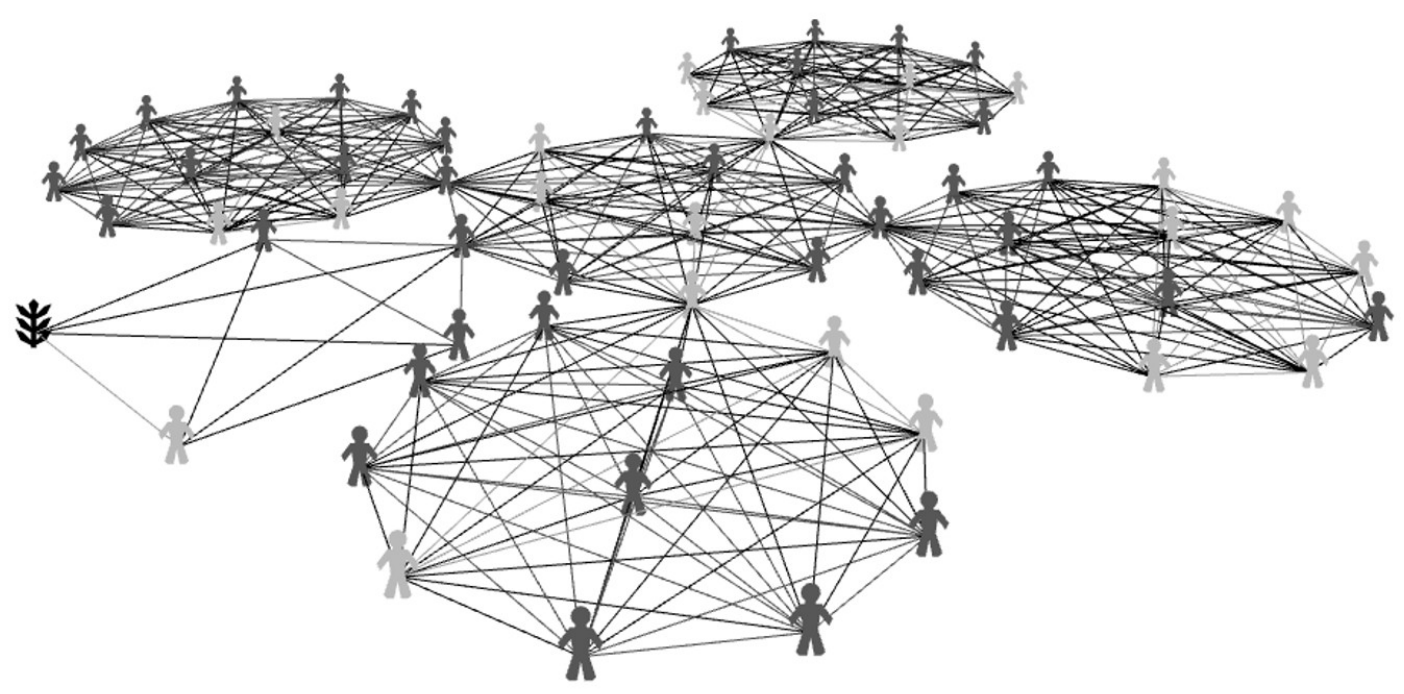

Note: Exemplary hierarchical network structure for Geblen. Dark grey agents belong to the cultural majority and light grey depicts the minority. The first adopter is plant-shaped and resides in a marginal position.

Figure 11: Preferential attachment network

Preferential attachment with marginal position of first adopter

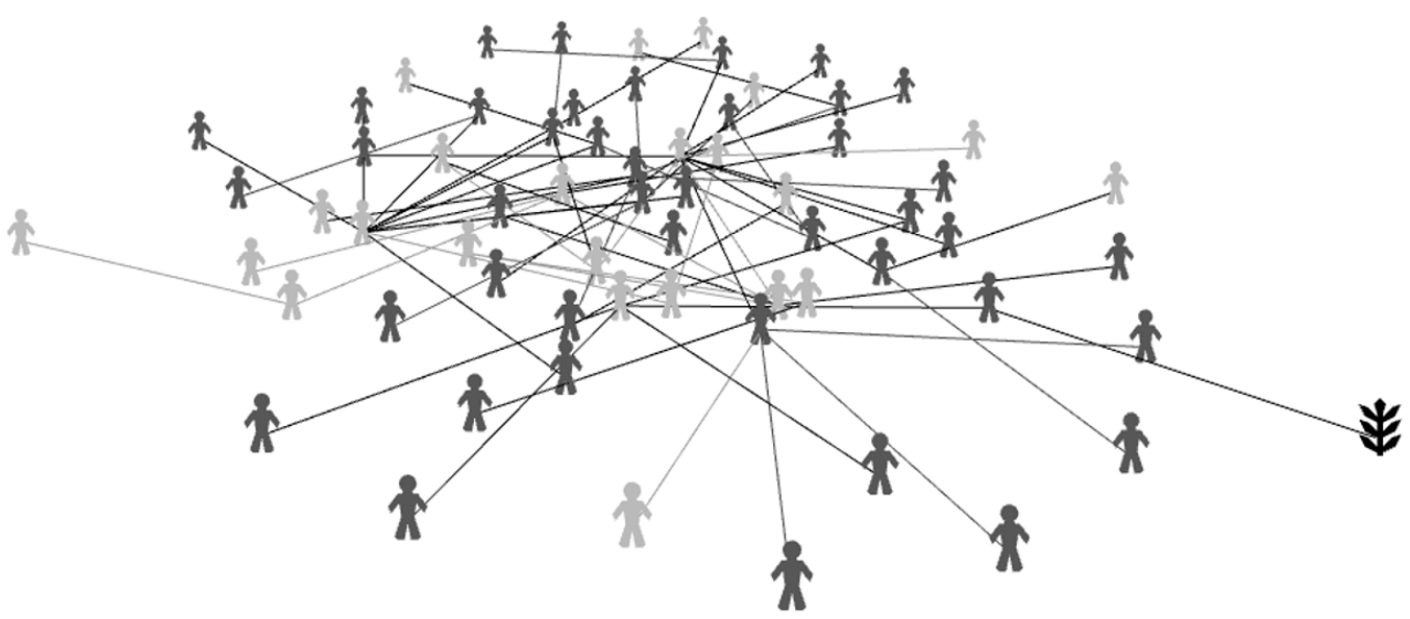

Note: Exemplary preferential attachment network structure for Geblen. Dark grey agents belong to the cultural majority and light grey depicts the minority. The first adopter is plant-shaped and resides in a marginal position. 
Figure 12: Small world with high clustering network

Small world with high clustering coefficient and marginal position of first adopter

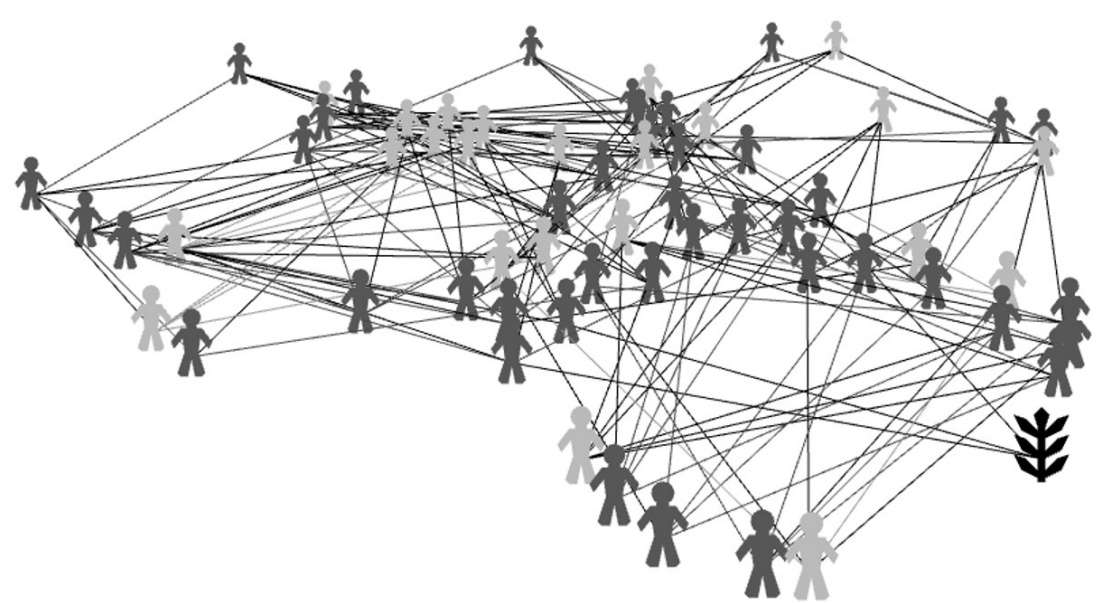

Note: Exemplary small world with high clustering network structure for Geblen. Dark grey agents belong to the cultural majority and light grey depicts the minority. The first adopter is plant-shaped and resides in a marginal position.

Figure 13: Small world with low clustering network

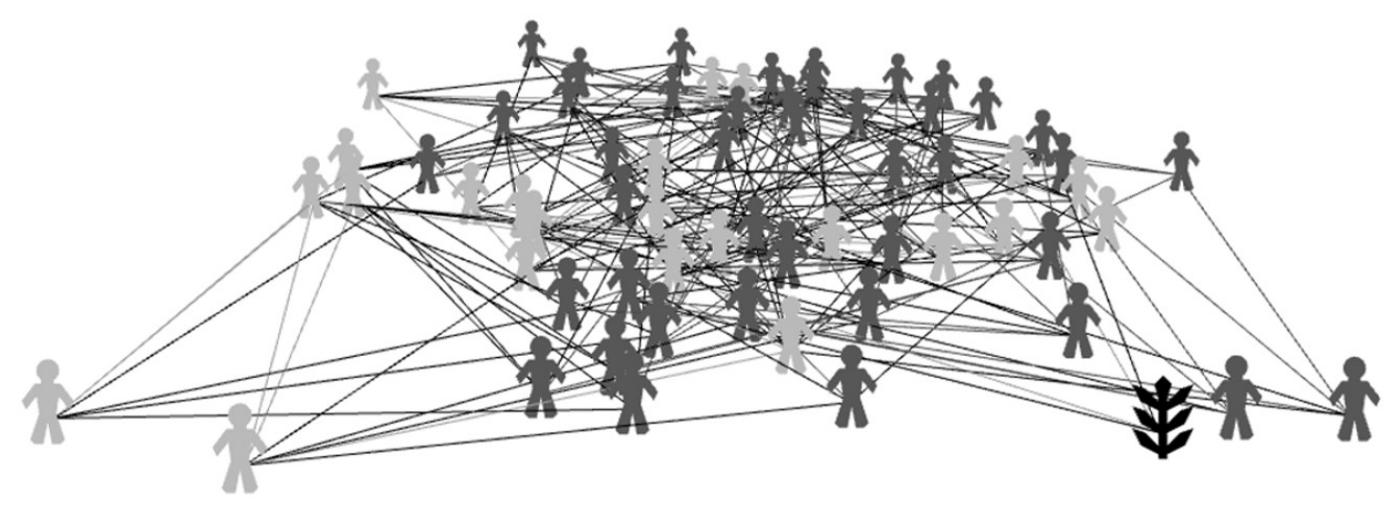

Note: Exemplary small world with low clustering network structure for Geblen. Dark grey agents belong to the cultural majority and light grey depicts the minority. The first adopter is plant-shaped and resides in a marginal position. 
Figure 14: Adado: Simulation results

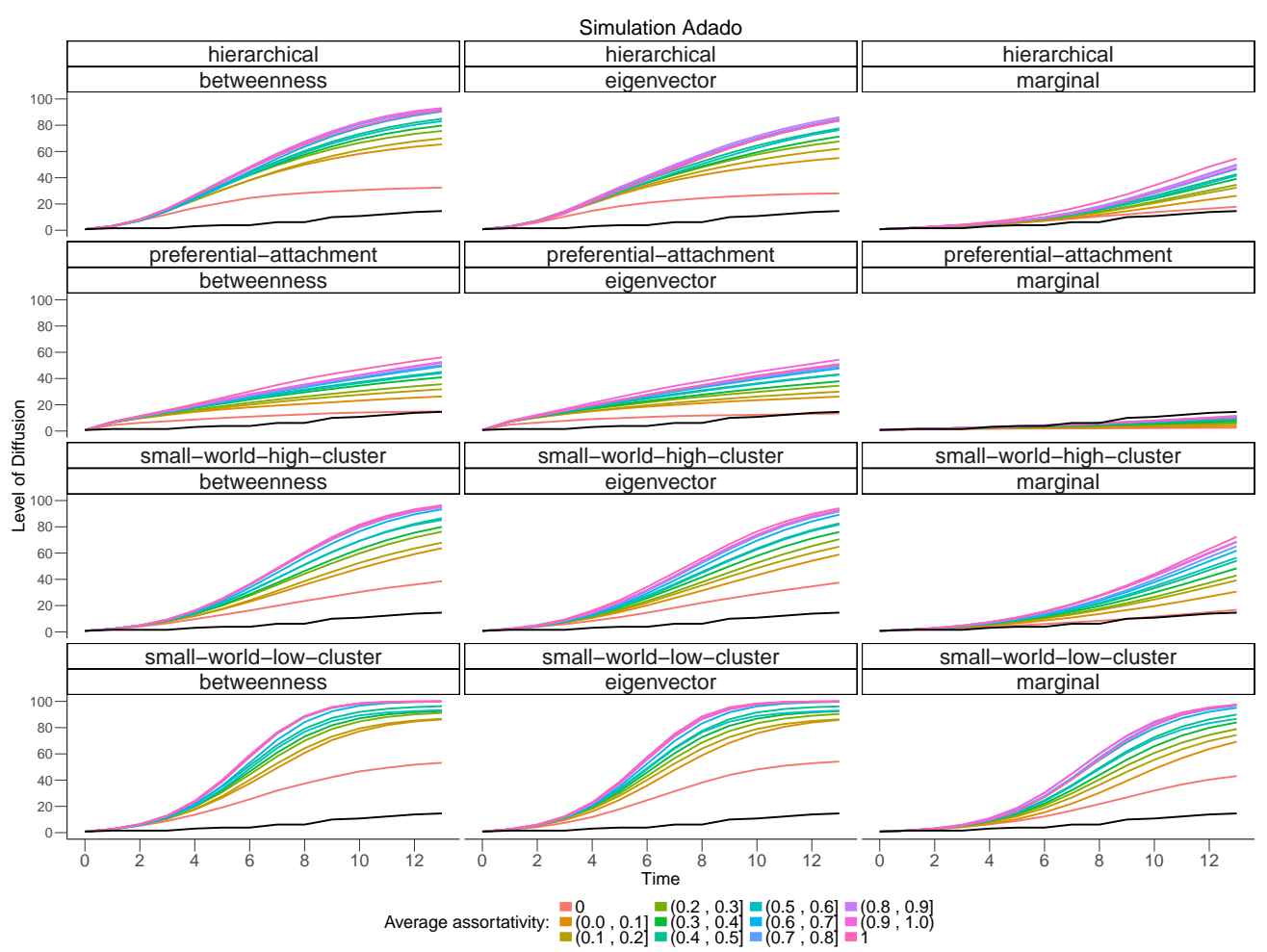

Source: Author's calculations based on ERHS.

Note: Simulation results for Adado split by type of network and position of the initial adopter. In each graph coloured lines represent diffusion by average assortativity of the system, while the black line depicts the true diffusion path of Adado.
Figure 15: Doma: Simulation results

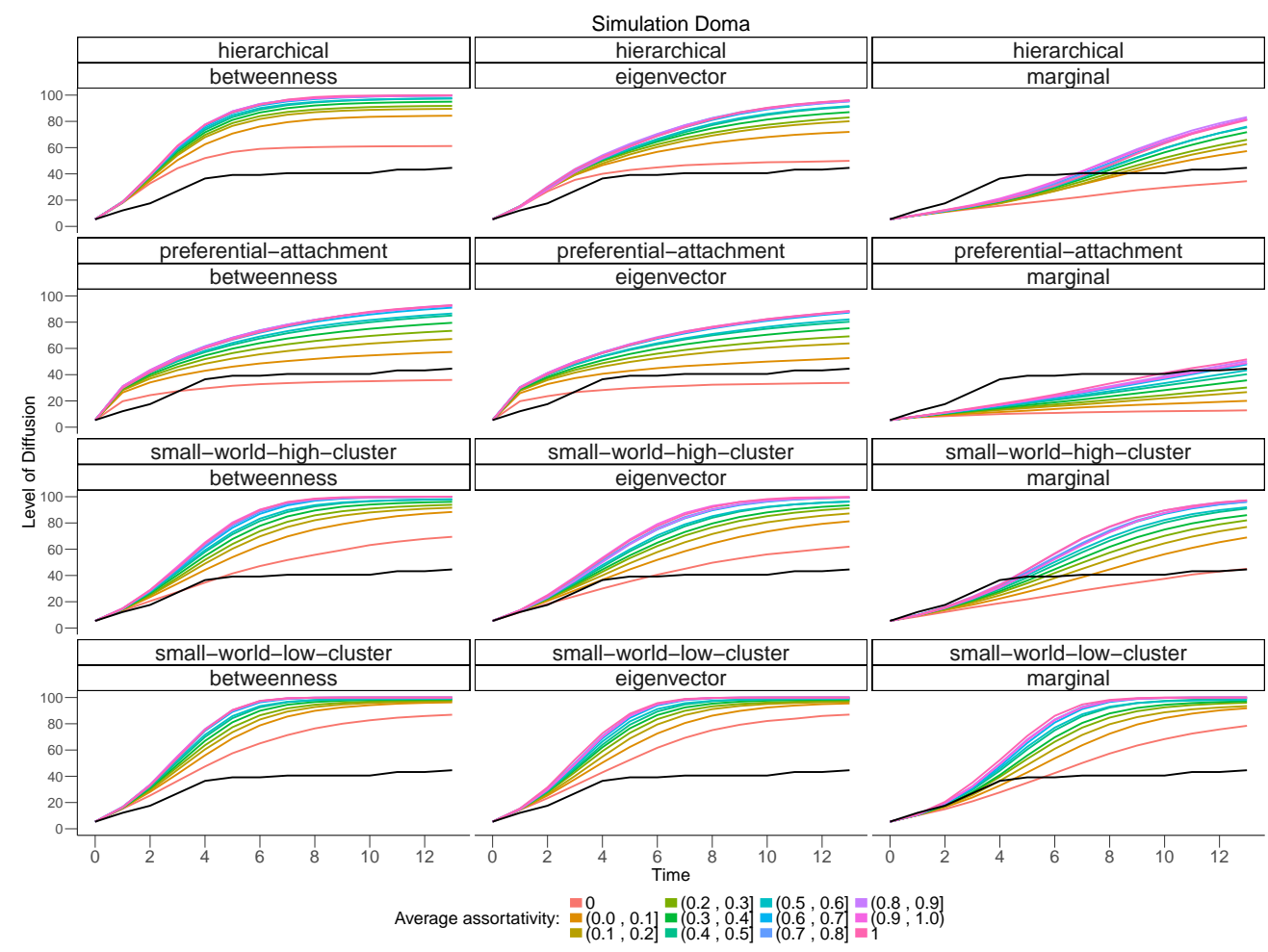

Source: Author's calculations based on ERHS.

Note: Simulation results for Doma split by type of network and position of the initial adopter. In each graph coloured lines represent diffusion by average assortativity of the system, while the black line depicts the true diffusion path of Doma. 
Figure 16: Geblen: Simulation results

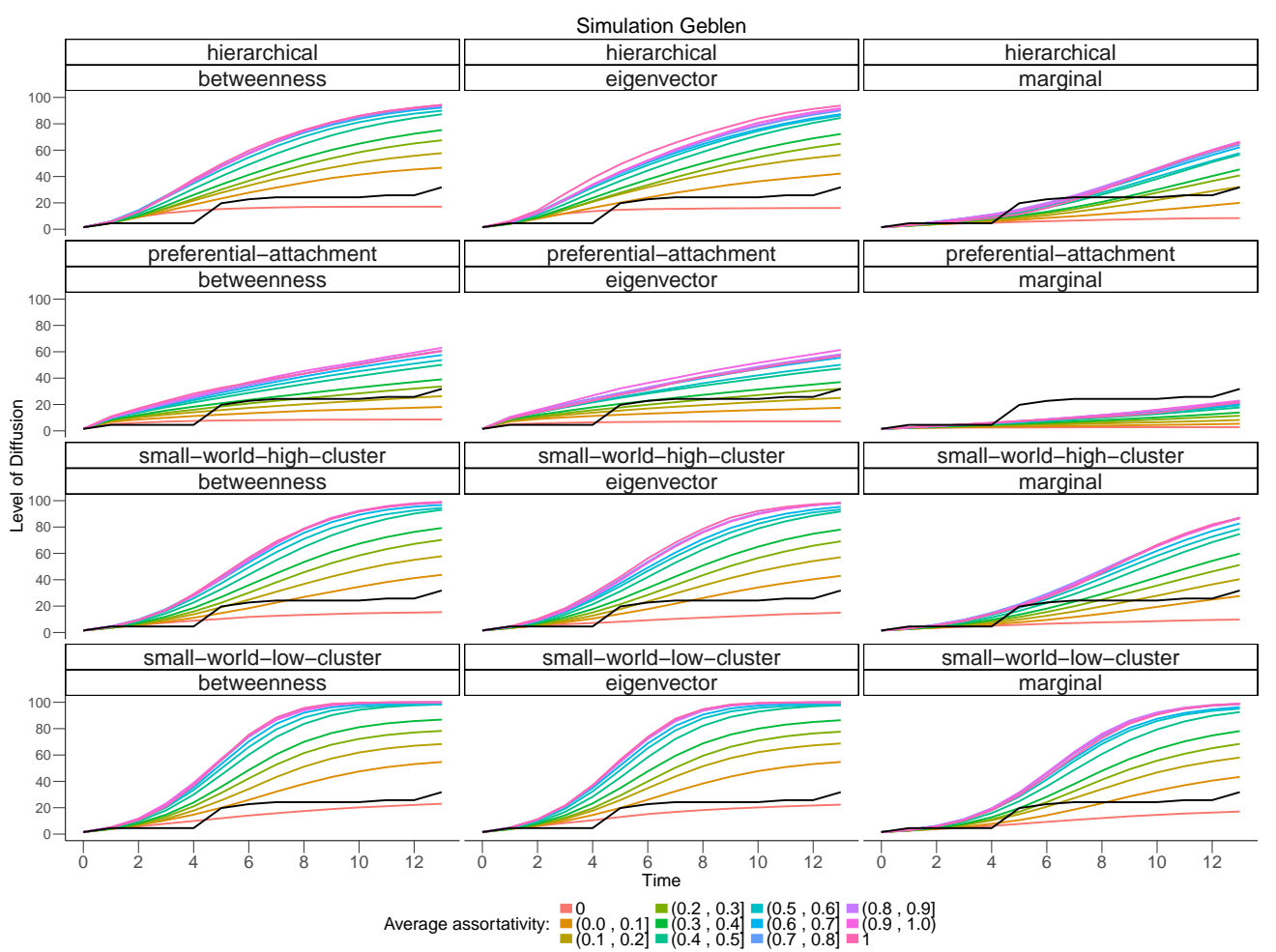

Source: Author's calculations based on ERHS.

Note: Simulation results for Geblen split by type of network and position of the initial adopter. In each graph coloured lines represent diffusion by average assortativity of the system, while the black line depicts the true diffusion path of Geblen.
Figure 17: Imdibir: Simulation results

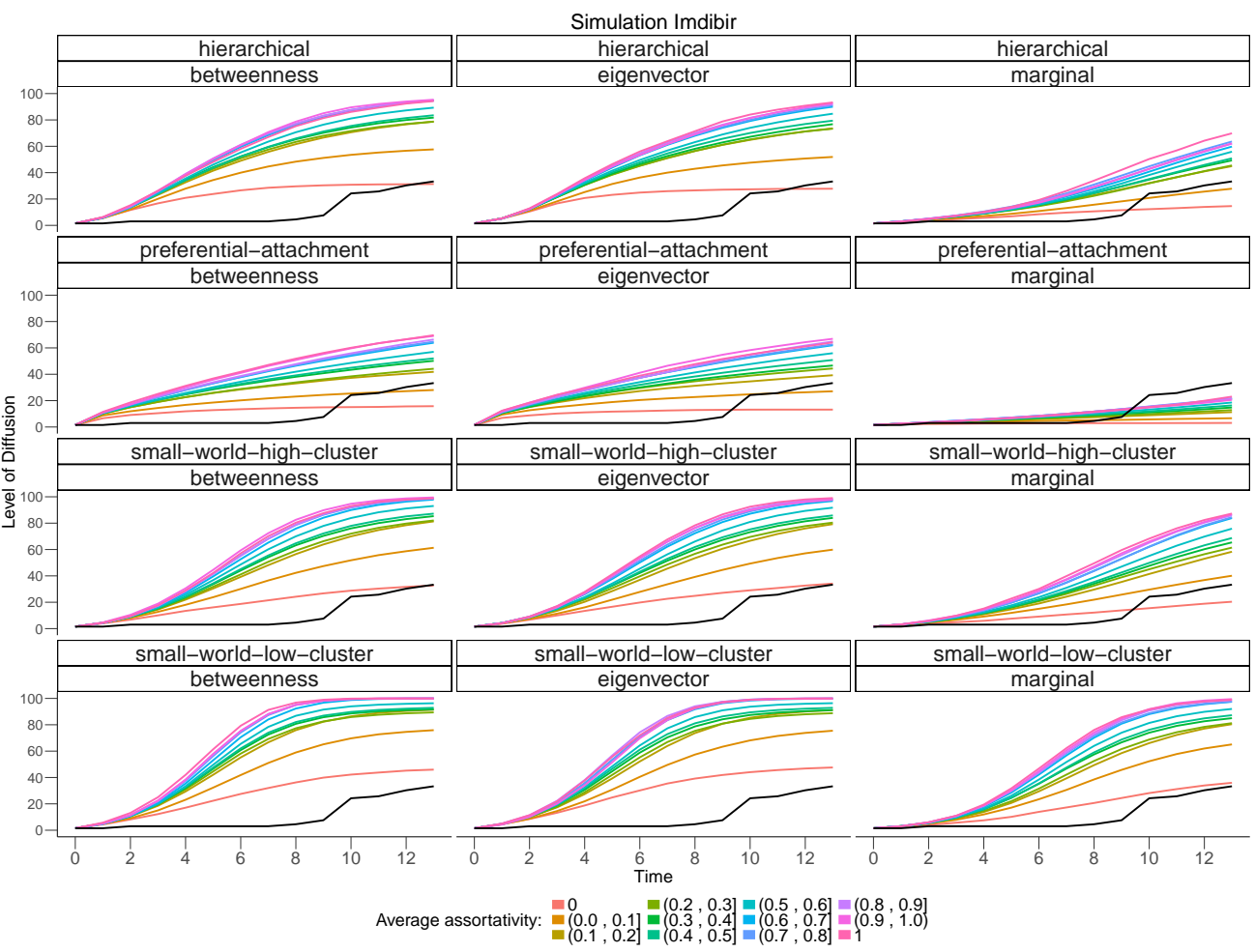

Source: Author's calculations based on ERHS.

Note: Simulation results for Imdibir split by type of network and position of the initial adopter. In each graph coloured lines represent diffusion by average assortativity of the system, while the black line depicts the true diffusion path of Imdibir. 
Figure 18: Shumsheha: Simulation results

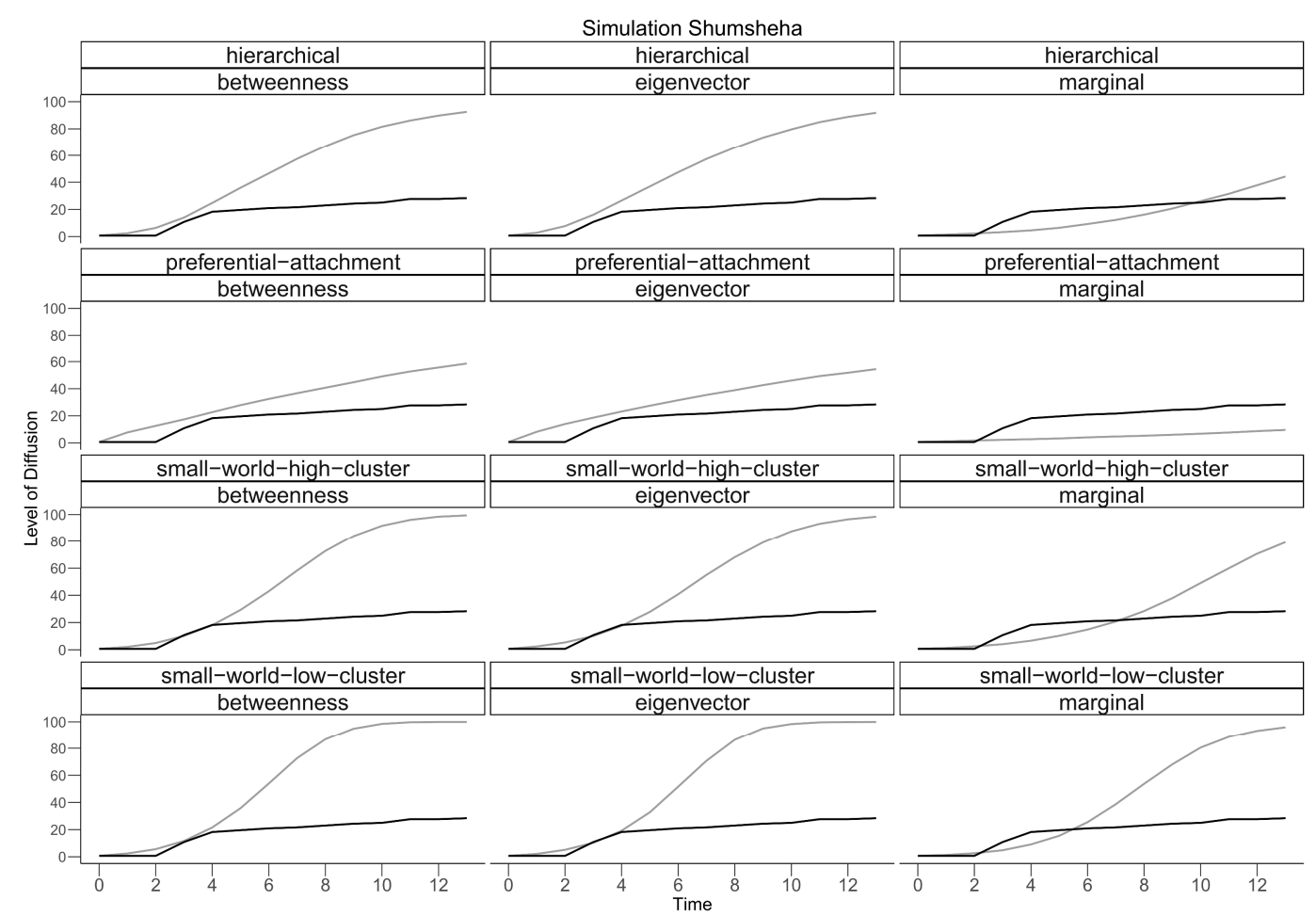

Source: Author's calculations based on ERHS

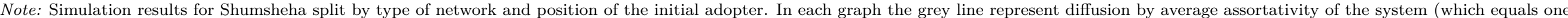
for all settings as no cultural distinctions prevail in Shumsheha), while the black line depicts the true diffusion path of Shumsheha. 